\title{
Rumor Spreading of an SIHR Model in Heterogeneous Networks Based on Probability Generating Function
}

\author{
Jinxian Li $\mathbb{D}^{1,}{ }^{1,2}$ Yanping $\mathrm{Hu},{ }^{1}$ and Zhen Jin $\mathbb{D}^{2,3}$ \\ ${ }^{1}$ School of Mathematical Sciences, Shanxi University, Taiyuan 030006, China \\ ${ }^{2}$ Shanxi Key Laboratory of Mathematical Techniques and Big Data Analysis on Disease Control and Prevention, \\ Taiyuan 030006, China \\ ${ }^{3}$ Complex System Research Center, Shanxi University, Taiyuan 030006, China
}

Correspondence should be addressed to Jinxian Li; lijinxian@sxu.edu.cn

Received 19 January 2019; Revised 2 May 2019; Accepted 27 May 2019; Published 23 June 2019

Academic Editor: Pietro De Lellis

Copyright (C) 2019 Jinxian Li et al. This is an open access article distributed under the Creative Commons Attribution License, which permits unrestricted use, distribution, and reproduction in any medium, provided the original work is properly cited.

This paper focuses on the modeling of a rumor spreading in heterogeneous networks. Using the probability generating function method and pair approximation method, the current research obtains nonlinear differential equations to describe the dynamics of rumor spreading. The comparison between numerical simulations and Monte Carlo simulations confirms the accuracy of our model. Furthermore, the threshold condition is also obtained in this paper. The numerical simulation results show that the heterogeneity of the network accelerates the outbreak of rumors but reduces the maximum density of spreader and the scale of rumors. The present study also examines the effects of parameters on rumor transmission and the differences between rumor transmission recovery mechanisms and disease transmission recovery mechanisms.

\section{Introduction}

Rumor is an important form of human communication. It can be interpreted as an infection of the mind, which is also defined as a typical social phenomenon which runs through the whole evolution of mankind [1]. Its spreading can cause damage to personal reputations, affect the financial markets, and cause social panic and instability $[2,3]$. Traditionally, rumors are propagated by word of mouth [4]. Nowadays, with the emergence of the Internet, people can spread rumors with instant messengers, emails or microblogs, and so on, which speeds up the dissemination of rumors virtually and makes rumors more influential than ever before [5]. The research on rumor propagation will offer us a thorough understanding of this issue and allow us to predict and reduce the possible harms caused by rumor propagation.

The study of the spread of the rumor began in the 1960s. Daley and Kendall [6,7] first proposed the classical DK model of rumor spreading in which the total population is divided into three groups: the people who are spreading the rumor (spreaders), those who have never heard the rumor (ignorants), and the ones who have heard the rumor but do not spread it (stiflers), and they denoted them as $S, I$, and $R$, respectively. Afterwards, Maki and Thomson [8] developed another classical MK model in 1973, which focused on the analysis of the rumor spreading based on mathematical theory via direct contact between spreaders and others. After that, more and more scholars paid attention to the spread of rumors. On one hand, more refined rumor spreading models are derived to model more detailed processes; on the other hand, the impacts of network structure on the rumor spreading are noticed.

To describe the process of rumor spread more carefully, the population is divided more finely. Xia et al. [9] extended the classic SIR rumor spreading model to a new SEIR rumor propagation model considering hesitating mechanism in complex social networks. Exposed (E) are those who have been infected, in the hesitating state, and do not spread rumors. In addition, in real life, there may be some people who do not agree with the rumor. They may persuade others to resist the rumor propagation. So Zan et al. [10] presented a susceptible-infected-counter-susceptible (SICS) 
rumor spreading model with counter mechanism in complex social networks. Then they derived mean-field equations to describe their dynamics in homogeneous networks and study the steady-state. In many cases, a rumor that concerns one event does not spread along, as a rumor cannot always spread without being affected by another rumor. To investigate the effects of interactions between two rumors, Jie et al. [11] built a model of a two-rumor interaction in which each individual has two states, which correspond to the attitudes toward each of the two rumors, and they built the corresponding ODE system. Gu et al. [12] revealed that the forgetting and remembering mechanism has great influence on the spread of rumors by extensive simulations. In view of this, Zhao et al. [13] added a new compartment, Hibernators, to the classic SIR model and they derived a new rumor spreading model, Ignorant-Spreader-Hibernator-Stifler (SIHR) model in homogeneous networks. They [13] investigated the final size of the rumor spreading under various spreading rates, stifling rates, forgetting rates, and average degrees of the network. All the above work studied rumor propagation in homogeneous networks, and the models were mean-field equations. Since the model loses a lot of information on the network, the threshold, below which the rumor final size is necessarily proportional to the fraction of initial speaker nodes but above which it is proportional to a fraction of the population, and the rumor final size may be missing in such case.

As early as in 1994, Lefevre and Picard [14] revealed the importance of network structure to rumor spreading. In fact, the topology of social networks shows highly complex connectivity in which each individual has a random number of connections to other individuals [15]. Zenette [16, 17] and Buvna et al. [18] performed a series of simulations in both static and small-world networks and showed the existence of a critical threshold of rumor spreading. So the influence of network structure on rumor spreading had been paid more and more attention to. Nekovee et al. [19] extended the former dynamic model to scale-free networks with assortative degree correlations and noticed the effect of degree correlation on rumor spreading. Moreno et al. [20] took into account that not only could nodes be in three different states $(S, I, R)$, but also they belong to different connectivity classes $k$ and built a mean-field equations which is degree-dependent. Zhao et al. [21] also extended their work in [13] to describe rumor propagation by means of the degree-dependent mean-field equation. See more similar work in [22-27].

Heterogeneity makes it difficult to derive differential equations to describe the epidemic. In fact, the heterogeneity of the network is not only reflected in the heterogeneity of degree distribution, but also in the locality of rumor spreading along the edge. So we try to find a better way to describe rumor spreading, rather than mean-field theory. Volz et al. [28] resolved this dilemma by showing the SIRepidemic dynamics with a system of three nonlinear ODE's which involves the PGF. See more similar work in $[29,30]$. In this work, we try to describe the rumor spreading in $[13,21]$ by the method based on PGF. There are still differences between rumor spreading and disease spreading mechanism. In the process of disease transmission, the recovery of the infected persons is not related to other nodes. The recovery is not dependent on the network structure. However, since the spreading wants to recover, he has to contact a node that is not an ignorant, so, the rumor recovery mechanism depends on the network structure. What is more, the population can move between the compartment of the hibernators and the spreaders in the rumor spreading. For these reasons, the establishment of rumor propagation model based on PGF encounters irresolvable difficulties. So we have to combine the PGF and the pair approximation method to model this process. Our results show that this method is indeed a better way to describe the rumor propagation. Then we also examine the effects of parameters on rumor transmission and the differences between rumor transmission recovery mechanisms and disease transmission recovery mechanisms.

The remainder of this paper is organized as follows. We derive the nonlinear ODE's model to describe the dynamics of the rumor spreading in Section 2. Furthermore, we obtain the rumor spreading threshold. In Section 3, we test this model in Poisson network and a refined scale-free network and compare the numerical simulations with Monte Carlo simulations, which shows that the numerical simulations of our model more consistently fit in with the Monte Carlo simulations. Then numerical simulations are conducted to further investigate the properties of parameters on the spread of the rumor. Finally, conclusions and discussions are given.

\section{SIHR Model in Random Networks}

This section introduces a low-dimensional system that models the percolation of an SIHR-rumor spreading in a heterogeneous network. It also obtains the disease thresholds.

2.1. Rumor Propagation Mechanism. This paper uses the rumor spreading rules proposed in Zhao et al. [13], which can be summarized as follows. (1) When a spreader contacts with an ignorant, the last one becomes a spreader with probability $\lambda$, namely, spreading rate. (2) When an ignorant contacts a spreader, the ignorant becomes a stifler with probability $\beta$ directly, namely, refusing rate. (3) The hibernators reflect the repeatability of rumor spreading. The spreaders could forget the rumor and then remember it. Spreaders spontaneously forget the rumor and switch their state to hibernators at the rate $\delta$ as a result of the forgetting mechanism, namely, forgetting rate. The remembering mechanism also exists for hibernators. Hibernators spontaneously remember the rumor and become the spreaders at the rate $\xi$ that is called spontaneous remembering rate. Besides, when a Hibernator contacts a spreader, the hibernator switches to be a spreader with probability $\eta$, namely, wakened remembering rate. (4) When a spreader contacts another spreader or a hibernator or a stifler, only the initiating spreader turns into a stifler with probability $\alpha$, namely, stifling rate.

When a rumor spreads through a network, the nodes can be in any of the exclusive states: ignorants $(\mathscr{I})$, spreaders $(\mathcal{S})$, hibernators $(\mathscr{H})$, and stiflers, $(\mathscr{R})$, respectively. We use $S, I, H$, and $R$ to denote the fraction of nodes in the sets $\mathscr{I}, \mathcal{S}, \mathscr{H}$, and $\mathscr{R}$, respectively. In addition, they satisfy the normalization 


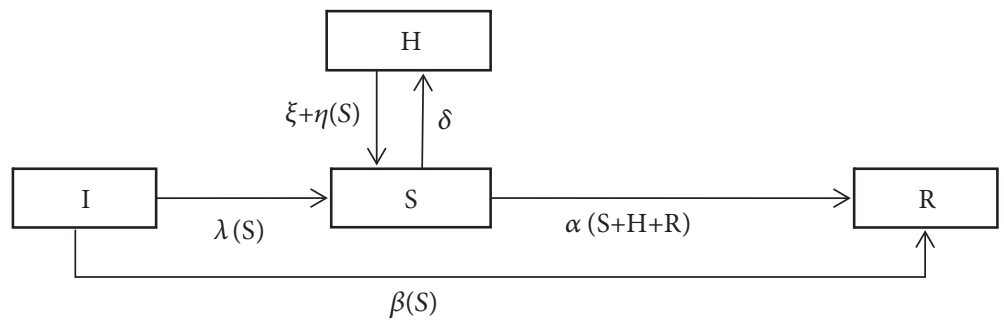

FIGURE 1: SIHR rumor spreading process.

condition: $S(t)+I(t)+H(t)+R(t)=1$. The SIHR rumor spreading process is shown in Figure 1.

2.2. Our Model. To model the above rumor spreading progress in a heterogeneous network, we consider a closed and mixed population $G=(V, E)$ with $\mathrm{N}$ individuals, where $V=\left\{v_{1}, v_{2} \cdots, v_{N}\right\}$ is the set of vertices representing the individuals and $E$ is the set of edges representing the connections among individuals. We also assume that contacts are symmetric, that is, if an edge $\left(v_{1}, v_{2}\right) \in E$ connects $v_{1}$ to $v_{2}$, then an edge also connects $v_{2}$ to $v_{1}$. Although the network is undirected (i.e., rumor can spread from each other), we wish to keep track of who spread. Therefore, for each edge $\left(v_{1}, v_{2}\right) \in$ $E$, we define two arcs as the ordered pairs $\left(v_{1}, v_{2}\right)$ and $\left(v_{2}, v_{1}\right)$. The first and second elements in the ordered pair $\left(v_{1}, v_{2}\right)$ are frequently called the ego and the alter, respectively [28]. Let $p_{k}$ be the degree distribution. Then $p(k)$ is the proportion of nodes having $k$ network contacts; i.e., $p(k)=[k] / N$, which can reveal the structure of this network. Then the probability generating functions (PGFs) of the degree distribution are given by

$$
g(x)=\sum_{k} p_{k} x^{k}
$$

Let $\bar{k}$ and $\overline{k^{2}}$ be the averaged degree and averaged secondmoment. Then they are $g^{\prime}(1)$ and $g^{\prime \prime}(1)+g^{\prime}(1)$, respectively.

Since the network is heterogeneous, we use $S_{k}(t), I_{k}(t)$, $H_{k}(t)$, and $R_{k}(t)$ to denote the density of spreaders, ignorants, hibernators, and stiflers with degree $\mathrm{k}$ at time $\mathrm{t}$, respectively. In addition, they satisfy the normalization condition: $S_{k}(t)+$ $I_{k}(t)+H_{k}(t)+R_{k}(t)=1$. Since our model is an extension of the network SIR model of Volz [28], we also need some notations in [28]. Let $\theta$ represent the fraction of degree one nodes that remain ignorant at time $t$ and $P_{A}^{B}$ represent the probability that an arc with an ego in state $A$ has an alter in state $B$, where $A$ and $B$ may be $I, S, H$, or $R$. Given $\theta$, it is easy to determine the fraction of nodes which remain ignorants at time t; i.e.,

$$
I=p_{0}+p_{1} \theta+p_{2} \theta^{2}+\cdots=g(\theta) \text {. }
$$

The model is formulated as follows:

$$
\begin{aligned}
\dot{\theta} & =-(\lambda+\beta) P_{I}^{S} \theta, \\
\dot{S_{k}} & =-\delta S_{k}+\xi H_{k}+\lambda k I_{k} P_{I}^{S}+\eta k H_{k} P_{H}^{S}
\end{aligned}
$$

$$
\begin{aligned}
& -\alpha\left(k S_{k}-k S_{k} \frac{\theta g^{\prime}(\theta) P_{I}^{S}}{\Sigma k p_{k} S_{k}}\right), \\
& \dot{H}_{k}=\delta S_{k}-\xi H_{k}-\eta k H_{k} P_{H}^{S} \text {, } \\
& \dot{P_{I}^{S}}=-(\lambda+\beta+\delta) P_{I}^{S}+\xi P_{I}^{H}+\lambda \frac{\theta g^{\prime \prime}(\theta) P_{I}^{I} P_{I}^{S}}{g^{\prime}(\theta)} \\
& +(\lambda+\beta)\left(P_{I}^{S}\right)^{2}+\eta \frac{\sum_{k} k(k-1) p_{k} H_{k}}{\sum_{k} k p_{k} H_{k}} P_{I}^{H} P_{H}^{S} \\
& -\alpha \frac{\Sigma_{k} k(k-1) p_{k} S_{k} P_{I}^{S}}{\Sigma_{k} k p_{k} S_{k}}\left(1-\frac{\theta g^{\prime}(\theta) P_{I}^{S}}{\Sigma_{k} k p_{k} S_{k}}\right), \\
& \dot{P}_{I}^{I}=(\lambda+\beta) P_{I}^{I} P_{I}^{S}-\frac{(\lambda+\beta) \theta g^{\prime \prime}(\theta) P_{I}^{I} P_{I}^{S}}{g^{\prime}(\theta)}, \\
& \dot{P_{I}^{H}}=-\xi P_{I}^{H}+\delta P_{I}^{S}+(\lambda+\beta) P_{I}^{S} P_{I}^{H} \\
& -\frac{\eta \Sigma_{k} k(k-1) p_{k} H_{k} P_{I}^{H} P_{H}^{S}}{\Sigma_{k} k p_{k} H_{k}}, \\
& \dot{P}_{H}^{S}=\delta \frac{\Sigma_{k} k p_{k} S_{k}\left(P_{S}^{S}-P_{H}^{S}\right)}{\Sigma_{k} k p_{k} H_{k}}+\xi P_{H}^{H}+\lambda \frac{\theta^{2} g^{\prime \prime}(\theta) P_{I}^{S} P_{I}^{H}}{\Sigma_{k} k p_{k} H_{k}} \\
& +\eta \frac{\Sigma_{k} k(k-1) p_{k} H_{k} P_{H}^{H} P_{H}^{S}}{\Sigma_{k} k p_{k} H_{k}}-(\delta+\eta+\alpha) P_{H}^{S} \\
& -\alpha \frac{\Sigma_{k} k(k-1) p_{k} S_{k} P_{H}^{S}}{\Sigma_{k} k p_{k} S_{k}}\left(1-\frac{\theta g^{\prime}(\theta) P_{I}^{S}}{\Sigma_{k} k p_{k} S_{k}}\right) \\
& +\eta\left(P_{H}^{S}\right)^{2}, \\
& \dot{P_{H}^{H}}=2 \delta P_{H}^{S}-\xi P_{H}^{H}-\eta \frac{\Sigma_{k} k(k-1) p_{k} H_{k} P_{H}^{H} P_{H}^{S}}{\Sigma_{k} k p_{k} H_{k}} \\
& -\delta \frac{\Sigma_{k} k p_{k} S_{k} p_{H}^{H}}{\sum_{k} k p_{k} H_{k}}+\eta P_{H}^{S} P_{H}^{H}, \\
& \dot{P}_{S}^{S}=(-\alpha-\delta) P_{S}^{S} \\
& +\frac{\Sigma_{k} k p_{k} H_{k}}{\sum_{k} k p_{k} S_{k}}\left(2 \xi P_{H}^{S}-\xi P_{S}^{S}+2 \eta P_{H}^{S}-\eta P_{H}^{S} P_{S}^{S}\right) \\
& +\lambda \frac{\theta^{2} g^{\prime \prime}(\theta)}{\sum_{k} k p_{k} S_{k}} P_{I}^{S}\left(2 P_{I}^{S}-P_{S}^{S}\right)
\end{aligned}
$$




$$
\begin{aligned}
& +\eta \frac{\sum_{k} k(k-1) p_{k} H_{k}}{\sum_{k} k p_{k} S_{k}} P_{H}^{S}\left(2 P_{H}^{S}-P_{S}^{S}\right) \\
& +\lambda \frac{\theta g^{\prime}(\theta)}{\sum_{k} k p_{k} S_{k}} P_{I}^{S}\left(2-P_{S}^{S}\right) \\
& -\alpha \frac{\sum_{k} k(k-1) p_{k} S_{k} P_{S}^{S}}{\sum_{k} k p_{k} S_{k}} \\
& +\alpha \frac{\theta g^{\prime}(\theta) \Sigma_{k} k(k-1) p_{k} S_{k} P_{S}^{S} P_{I}^{S}}{\left(\Sigma_{k} k p_{k} S_{k}\right)^{2}} \\
& -\alpha \frac{\theta g^{\prime}(\theta) P_{I}^{S} P_{S}^{S}}{\sum_{k} k p_{k} S_{k}} .
\end{aligned}
$$

It should be noted that since the spreaders and the hibernators can be transformed into each other, the model cannot be established by simply using the methods in the literature [28], which is the difficulty of the modeling process. The modeling process is given in detail in Appendix.

2.3. Initial Conditions and Spreading Threshold. If the probability of a node with degree $k$ selected uniformly at random and initial spreader is $\epsilon_{k}$, we can anticipate the following initial condition.

(1) $S_{k}=\epsilon_{k} N_{k} / N_{k}=\epsilon_{k}, M_{S}=\Sigma k p_{k} \epsilon_{k} / \Sigma k p_{k}, M_{I S} \approx M_{S}=$ $\Sigma k p_{k} \epsilon_{k} / \Sigma k p_{k}$.

(2) $M_{I}=1-M_{I S}=1-\Sigma k p_{k} \epsilon_{k} / \Sigma k p_{k}, M_{I I}=M_{I}-M_{I S}=$ $1-2 \Sigma k p_{k} \epsilon_{k} / \Sigma k p_{k}$.

(3) $P_{I}^{S}(t=0)=M_{I S} / M_{I}=\Sigma k p_{k} \epsilon_{k} /\left(\Sigma k p_{k}-\Sigma k p_{k} \epsilon_{k}\right)$, $P_{I}^{I}(t=0)=M_{I I} / M_{I}=\left(\Sigma k p_{k}-2 \Sigma k p_{k} \epsilon_{k}\right) /\left(\Sigma k p_{k}-\right.$ $\left.\sum k p_{k} \epsilon_{k}\right)$.

(4) $H_{k}=0, P_{I}^{H}=0$ (because there is no hibernator initially).

(5) $\theta \approx 1$, which can be interpreted as the fraction of nodes remaining ignorant.

Applying the initial conditions above into the $\dot{P}_{I}^{S}$ and considering $\epsilon_{k} \ll 1$ give

$$
\begin{aligned}
& \dot{P_{I}^{S}}(t=0) \\
& \approx-(\lambda+\beta+\delta) \frac{\Sigma k p_{k} \epsilon_{k}}{\Sigma k p_{k}-\Sigma k p_{k} \epsilon_{k}} \\
& +\frac{\lambda g^{\prime \prime}(1)}{g^{\prime}(1)} \frac{\Sigma k p_{k} \epsilon_{k}}{\Sigma k p_{k}-\Sigma k p_{k} \epsilon_{k}} \frac{\Sigma k p_{k}-2 \Sigma k p_{k} \epsilon_{k}}{\Sigma k p_{k}-\Sigma k p_{k} \epsilon_{k}} \\
& +(\lambda+\beta)\left(\frac{\Sigma k p_{k} \epsilon_{k}}{\Sigma k p_{k}-\Sigma k p_{k} \epsilon_{k}}\right)^{2} \\
& -\alpha \frac{\Sigma k(k-1) p_{k} \epsilon_{k}}{\Sigma k p_{k}-\Sigma k p_{k} \epsilon_{k}}+\alpha g^{\prime}(1) \frac{\Sigma k(k-1) p_{k} \epsilon_{k}}{\left(\Sigma k p_{k}-\Sigma k p_{k} \epsilon_{k}\right)^{2}} \text {. }
\end{aligned}
$$

For (4), giving up the higher order items gets

$$
\begin{aligned}
\dot{P_{I}^{S}}(t=0) \approx & -(\lambda+\beta+\delta) \frac{\sum k p_{k} \epsilon_{k}}{\sum k p_{k}}+\frac{\lambda g^{\prime \prime}(1)}{g^{\prime}(1)} \frac{\sum k p_{k} \epsilon_{k}}{\sum k p_{k}} \\
& -\alpha \frac{\sum k(k-1) p_{k} \epsilon_{k}}{g^{\prime}(1)}+\alpha \frac{\sum k(k-1) p_{k} \epsilon_{k}}{g^{\prime}(1)} \\
= & {\left[\frac{\lambda g^{\prime \prime}(1)}{g^{\prime}(1)}-(\lambda+\beta+\delta)\right] \frac{\sum k p_{k} \epsilon_{k}}{g^{\prime}(1)} . }
\end{aligned}
$$

Because $\Sigma k p_{k} \epsilon_{k} / g^{\prime}(1)>0$, the condition to guarantee $P_{I}^{S}>0$ is

$$
\frac{\lambda g^{\prime \prime}(1)}{g^{\prime}(1)}-(\lambda+\beta+\delta)>0 \text {. }
$$

Therefore, the threshold is

$$
\lambda_{c}=(\beta+\delta) \frac{g^{\prime}(1)}{g^{\prime \prime}(1)-g^{\prime}(1)}
$$

On the basis of above theoretical analysis, a nonzero rumor diffusion threshold $\lambda_{c}$ would be present. If $\lambda>$ $\lambda_{c}$, the rumor diffuses and necessarily occupies a fraction of population even as $\epsilon_{k} \longrightarrow 0$. And the rumor only spreads an infinitesimally small number of spreaders which approximates to zero compared with large population while $\lambda$ is below threshold $\lambda_{c}$. Next, take different values for $\epsilon_{k}$ as $\epsilon_{k}=\epsilon, \epsilon_{k}=\epsilon^{k}$ and $\epsilon_{k}=\epsilon^{1 / k}$. Equation (5) shows that the choice of $\epsilon_{k}$ does not affect the outbreak of the rumor. However,

$$
\left.\dot{P}\right|_{\epsilon_{k}=\epsilon^{1 / k}}>\left.\dot{P}\right|_{\epsilon_{k}=\epsilon}>\left.\dot{P} S\right|_{\epsilon_{k}=\epsilon^{k}} .
$$

Hence, the selection of initial spreader nodes will not affect the threshold, but will affect the speed of rumor expansion.

We should also note that there is a threshold for rumor spreading in this paper, which is absent in the literature [13, 21]. Furthermore, the threshold depends not only on averaged degree $g^{\prime}(1)$ but also on the fluctuations of the connectivity distribution $g^{\prime \prime}(1)$.

\section{Numerical Simulations and Monte Carlo Simulations}

In this section, we carry out Monte Carlo simulations and numerical simulations to verify the above analytical results and further investigate the effects of parameters on the rumor spreading model. For Monte Carlo $N=10^{4}$. The detailed process can be seen in [31]. If no otherwise specified, the numerical simulations of model (3) are performed with $N=$ $10^{4}$. Since model (3) involves $S_{k}$ and $H_{k},(k=1,2, \ldots)$, to simplify the problem, we truncated the network to a maximum degree of 30 . The initial condition of the numerical simulations of the model is $\epsilon=10^{-4}$. For the sake of conveniences, we define the cumulative rumor incidence as the fraction of nodes spreaders, stiflers, or hibernators. The 


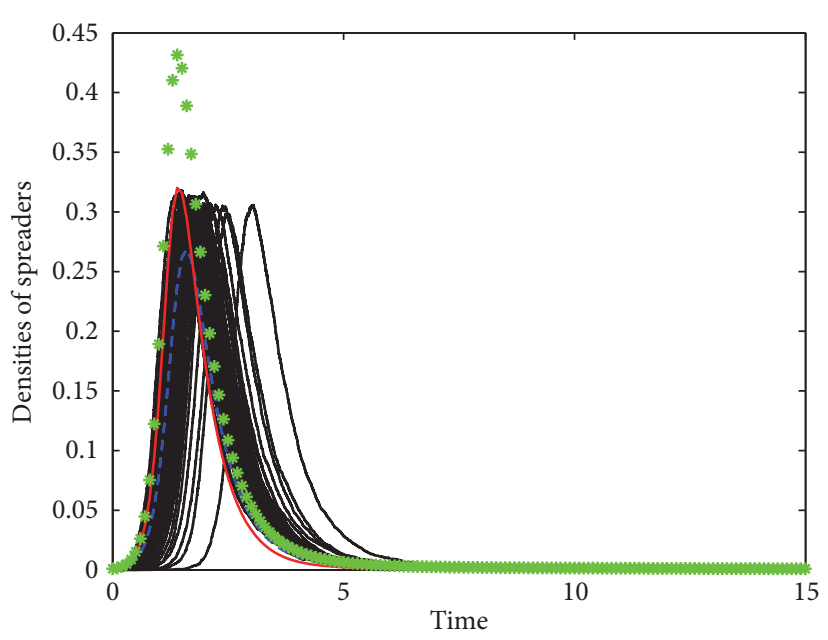

(a)

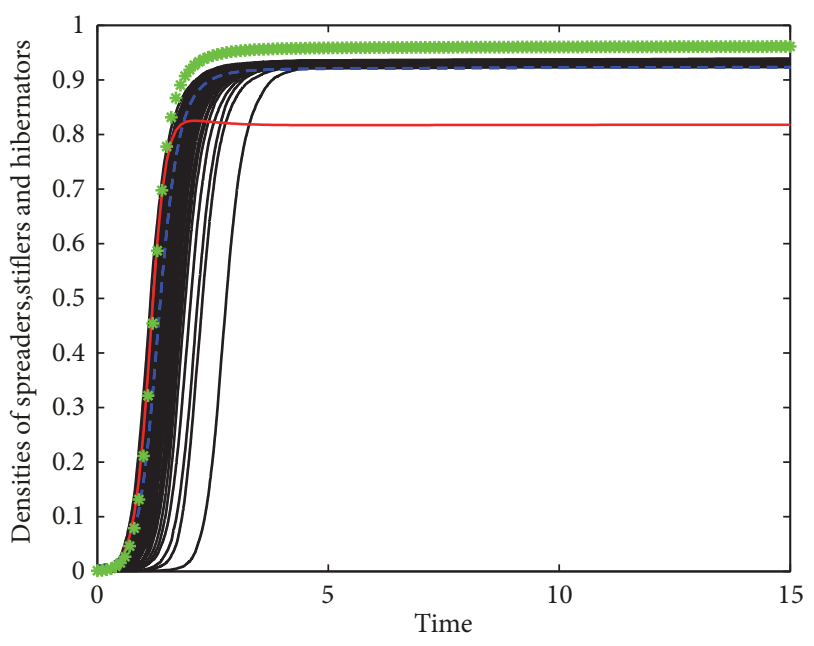

(b)

Figure 2: Simulations with $\lambda=0.7, \beta=0.3, \alpha=0.2, \delta=0.1, \xi=0.1$, and $\eta=0.2$. The black lines correspond to 100 Monte Carlo simulations for an SIHR rumor model in a network with the Poisson degree distribution with $\bar{k}=10$. The red solid lines and green star lines represent numerical simulation trajectories based on the mean-field system in [13] and [21], respectively. The blue dashed lines represent numerical simulation trajectories based on system (3). (a) The trajectories of the density of spreaders. (b) The trajectories of the densities of spreaders, stiflers, and hibernators.

peak value of spreader density $\max \{S(t)\}$ can be used to measure the maximum rumor influence.

In Figure 2, the black solid lines show the result of 100 Monte Carlo simulations for an SIHR rumor model in a network with the Poisson degree distribution with $\bar{k}=10$. The red solid lines and green star lines represent numerical simulation trajectories based on the mean-field systems in [13] and [21], respectively. The blue dashed lines represent numerical simulation trajectories based on system (3). Figure 2(a) exhibits the trajectories of the density of spreaders and (b) exhibits the cumulative rumor incidence (densities of spreaders, stiflers and hibernators). And obviously, the blue dashed lines which cut through the dense mass of simulation trajectories are the analytical trajectories based on model (3). This implies that the model of (3) can well describe the spread of rumors in complex networks.

In order to further verify the reliability of model (3), we perform Monte Carlo simulations and numerical simulations of model (3) in a refined Power-law network. The network considered here can be generated by assigning a random number of lines to a set of $N$ nodes according to the distribution $p_{k}$, where

$$
p_{k}= \begin{cases}1-\sum_{k=2}^{30}\left(\frac{43}{90}\right) k^{(-1.2)}, & k=1, \\ \left(\frac{43}{90}\right) k^{(-1.2)}, & k=2,3, \ldots, 30 .\end{cases}
$$

Then by the Configuration Model (CM) process [31], we can get a new network which is shown to have characteristic of Power-law network in Figure 3. Figure 3 shows the degree distribution of this new network in double logarithmic coordinates system.

In Figure 4, the Monte Carlo simulations for the network with the refined Power-law degree distribution in Figure 3 and the numerical simulation of model (3) and the meanfield system in $[13,21]$ are performed with $N=10^{4}$ and $p_{k}$ in Figure 3. The black solid lines show the results of 100 Monte Carlo simulations for the Power-law network. The red solid lines and green star lines represent numerical simulation trajectories based on the mean-field systems in [13] and [21], respectively. The blue dashed lines represent a numerical simulation trajectories based on model (3). Obviously, the blue lines which nearly cut through the dense mass of simulation trajectories are the analytical trajectories based on system (3). This implies that the model of (3) can well describe the spread of rumors in complex networks.

Figure 5 compares the spread of SIHR rumors in networks with the Poisson degree distribution and in the network with the refined power-law degree distribution. The results show that the final scale of rumors in the network with the Power-law degree distribution is smaller than that with the Poisson degree distribution. Furthermore, the density of spreaders reaches its maximum earlier, which is smaller than that with the Poisson degree distribution. Although heterogeneity accelerates the spread of rumors, it reduces the scale of spread.

The simulation results in Figure 6 show the evolution of rumor diffusion over time with different spreading rates $\lambda=$ $0.2, \lambda=0.4$, and $\lambda=0.5$, respectively. In Figure 6(a), it is clear to see that the densities of the spreaders have sharp increases until they reach their peaks and then decrease to zero. Meanwhile, we can see that, with the increase of the spreading rate $\lambda$, the density of spreaders reaches its maximum earlier and larger. Figure 6(b) shows the cumulative rumor incidence increases with the increase of spreading rate $\lambda$.

Figure 7 displays how the evolution of rumor diffusion changes over time under different refusing rates $\beta$, where we set $\beta=0.1, \beta=0.2$, and $\beta=0.4$, respectively. In Figure 7(a), it 


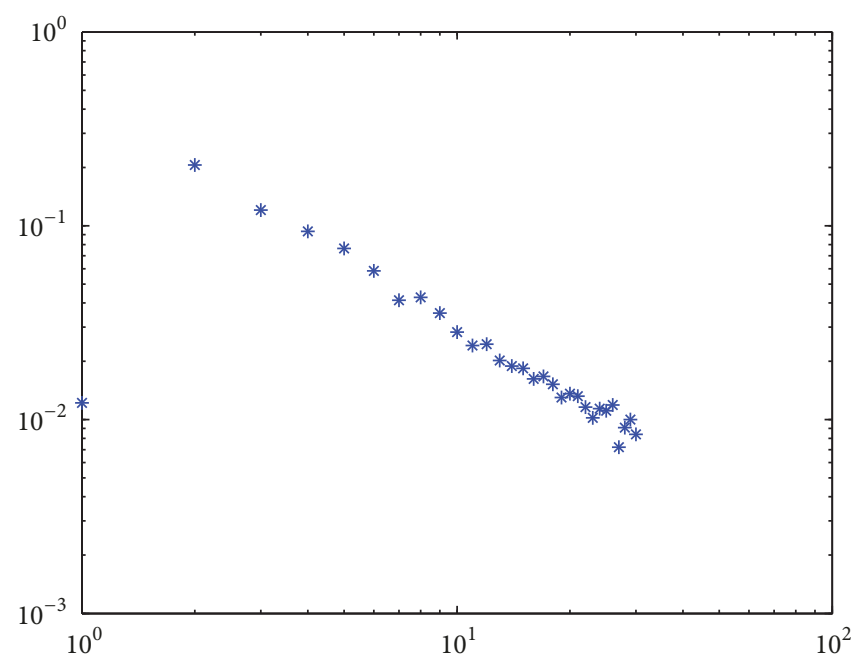

FIGURE 3: Degree distribution of refined power-law network.

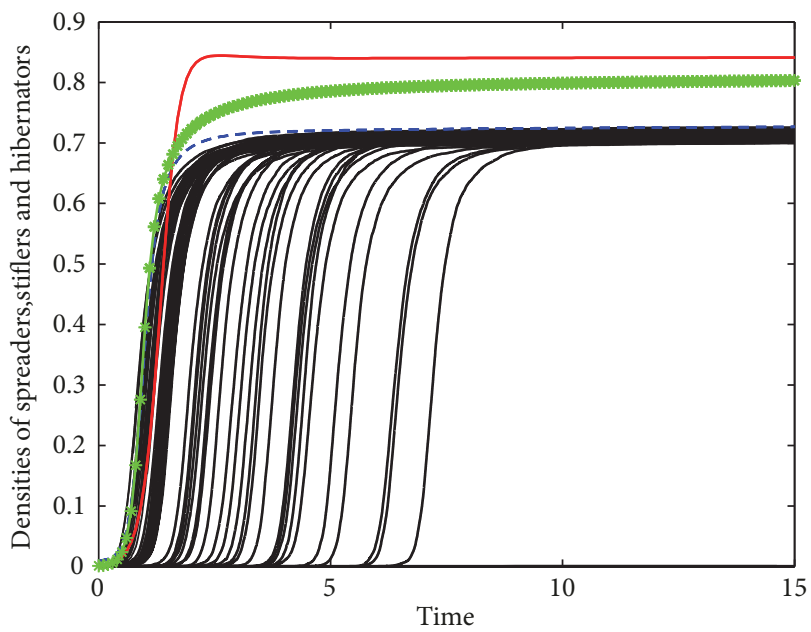

(a)

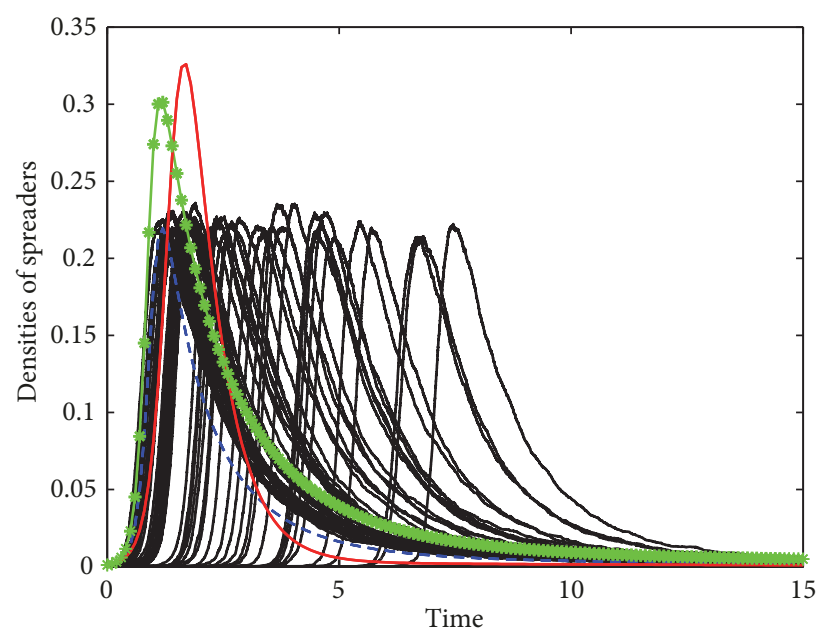

(b)

Figure 4: Simulations with $\lambda=0.7, \beta=0.3, \alpha=0.2, \delta=0.1, \xi=0.1$, and $\eta=0.1$. The black lines correspond to 100 Monte Carlo simulations trajectories for an SIHR rumor model in a network with the refined power-law degree distribution. The red solid lines and green star lines represent numerical simulation trajectories based on the mean-field system in [13] and [21], respectively. The dashed blue lines represent numerical simulation trajectories based on the system (3). (a) The trajectories of the densities of spreaders. (b) The trajectories of the densities of spreaders, stiflers, and hibernators.

is clear to see that the maximum of spreader density decreases with the increase of refusing rate $\beta$. If more people have the ability to see through the rumor or do not spread rumor (the direct link from the ignorants to the stiflers), the maximum rumor influence would become smaller. Figure 7 (b) shows the cumulative rumor incidence decreases with the increase of refusing rate $\beta$.

Figure 8 illustrates how the evolution of rumor diffusion changes over time under different stifling rates $\alpha$, where we set $\alpha=0.1, \alpha=0.2, \alpha=0.3$, and $\alpha=0.4$, respectively. Figure 8 shows that both the peak value of the spreader and the cumulative rumor incidence decrease with the increase of stifling rate $\alpha$ when $\alpha$ is small, while they increase with the increase of $\alpha$ when $\alpha$ is bigger. However, in this modeling process, we assume that the probability of a spreader node with $\mathrm{m}$ nonignorant neighbors recovered in unit time is $m \alpha$ if the stifling rate is $\alpha$, but, actually, it should be $1-(1-\alpha)^{m}$. That is, we use the following approximation: $1-(1-\alpha)^{m} \approx m \alpha$, which is obviously unreliable with larger $\alpha$.

From Figure 9, we can see that the maximum of the density of the spreader decreases with the increase of the forgetting rate $\delta$ and it has almost no effects on the cumulative rumor incidence. Figures 10 and 11 show that the remembering rate $\xi$ and the wakened remembering rate $\eta$ have weak effects on the rumor spreading process. From Figure 11(a), we can see that the peak value of the spreader density increases with the increase of the wakened remembering rate $\eta$. 


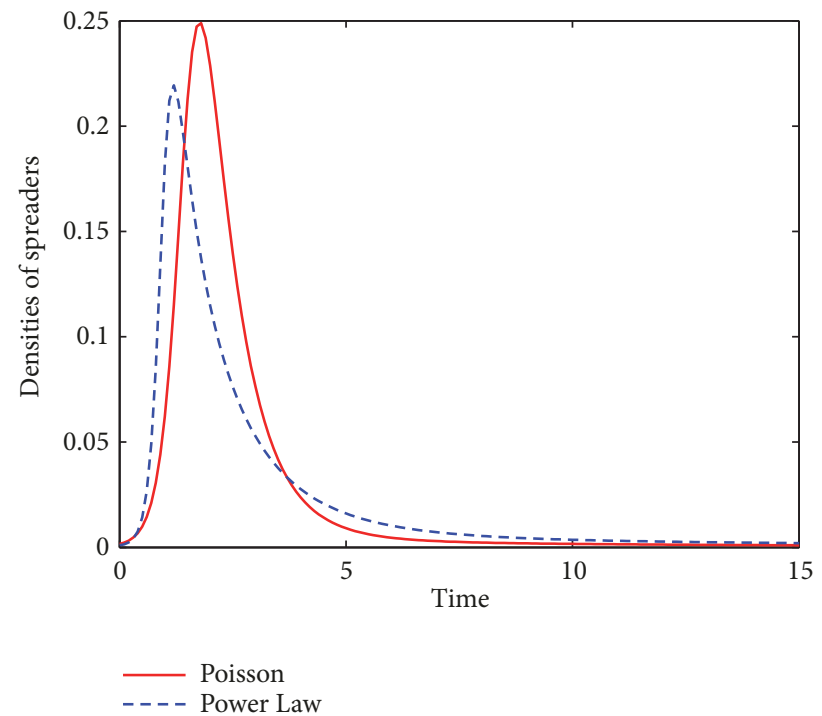

(a)

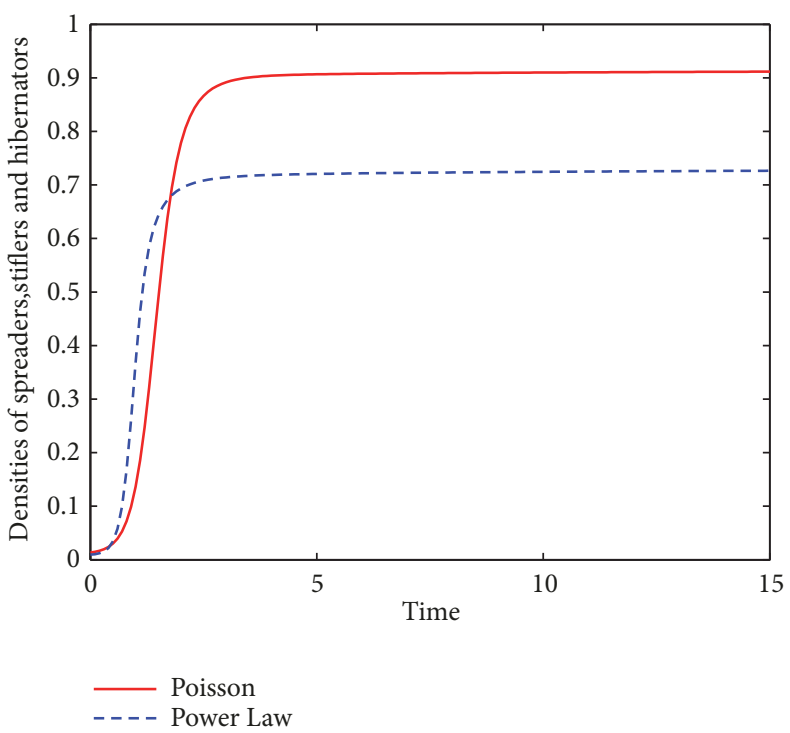

(b)

FiguRE 5: Numerical simulations of (3) with $\lambda=0.7, \beta=0.3, \alpha=0.2, \delta=0.1, \xi=0.1, \eta=0.1$ in the networks with different degree distributions but with the same averaged degree. The red solid lines and the blue dashed lines correspond to numerical simulations trajectories for an SIHR rumor model (3) in a network with the Poisson degree distribution and the refined power-law degree distribution, respectively. (a) The trajectories of the densities of spreaders. (b) The trajectories of the densities of spreaders, stiflers, and hibernators.

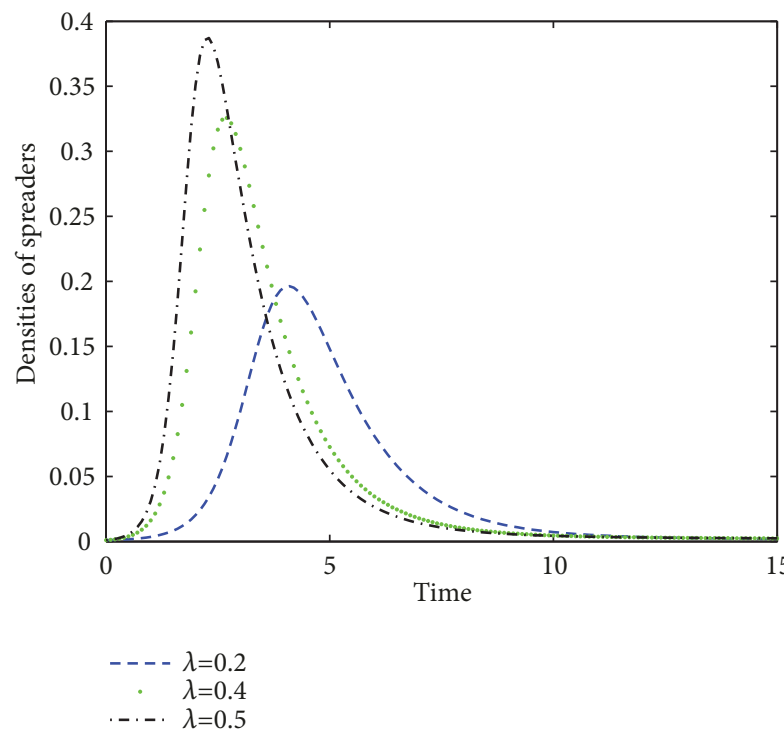

(a)

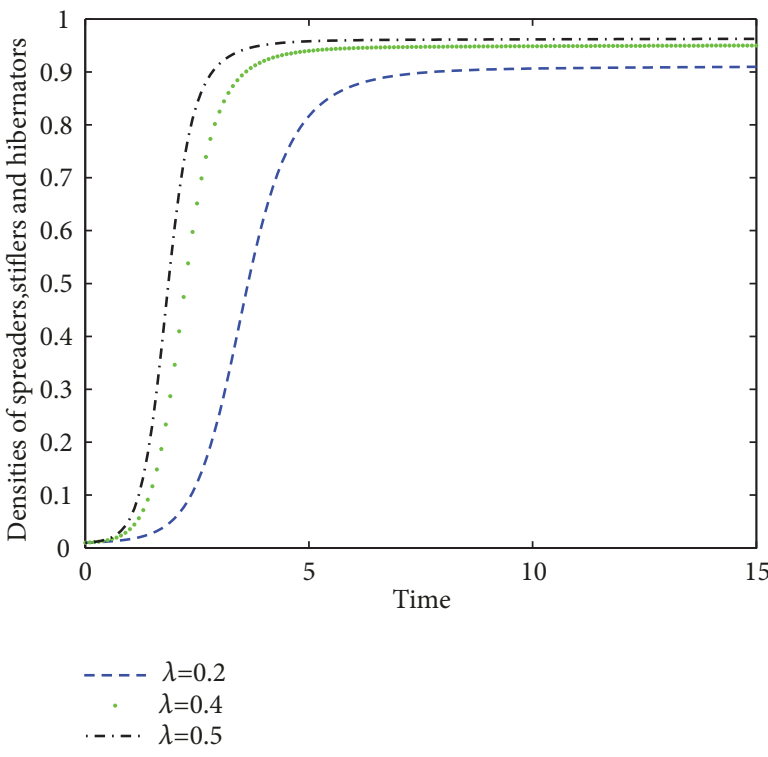

(b)

Figure 6: Numerical simulations of (3) with $\beta=0.1, \alpha=0.1, \delta=0.1, \xi=0.05$, and $\eta=0.1$ and different spreading rates $\lambda$ in the network with the Poisson degree distribution. (a) The trajectories of the densities of spreaders. (b) The trajectories of the densities of spreaders, stiflers, and hibernators.

Although the rumor spreading in social networks is similar to the disease spreading in biological networks, there are still differences between them. In the process of disease transmission, the recovery mechanism of the infected persons depends on themselves. However, a spreader turns into a stifler that needs to meet other spreaders, hibernators, or stiflers. So the recovery of spreader relies on the structure of network in the rumor spreading model. In order to compare the difference between the two mechanisms, we do the numerical simulations of SIHR model under two mechanisms, which are depending on the structure of network and depending on themselves. In Figure 12, we can see that the maximum rumor influence and the cumulative rumor incidence are lower under the mechanism depending 


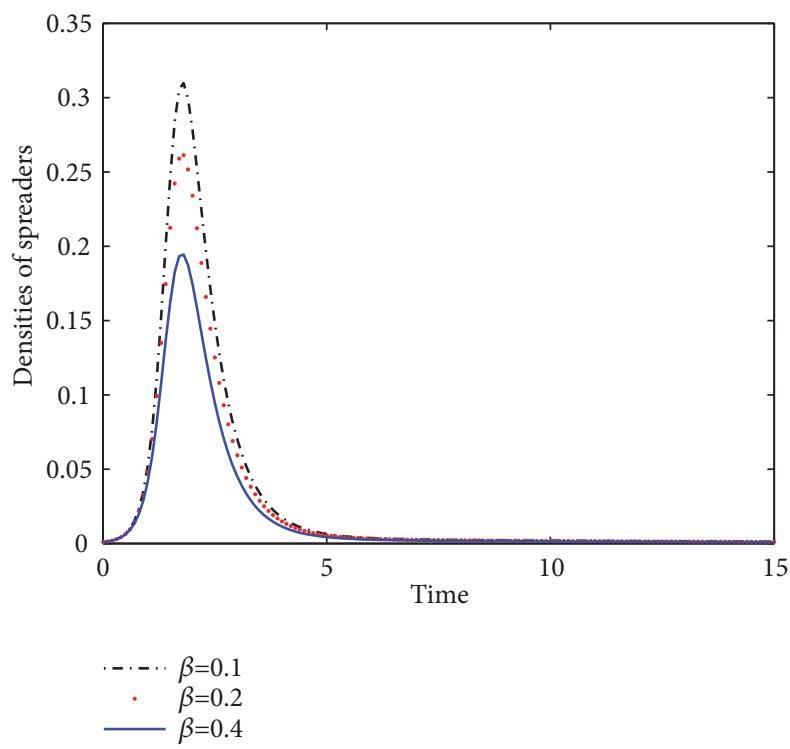

(a)

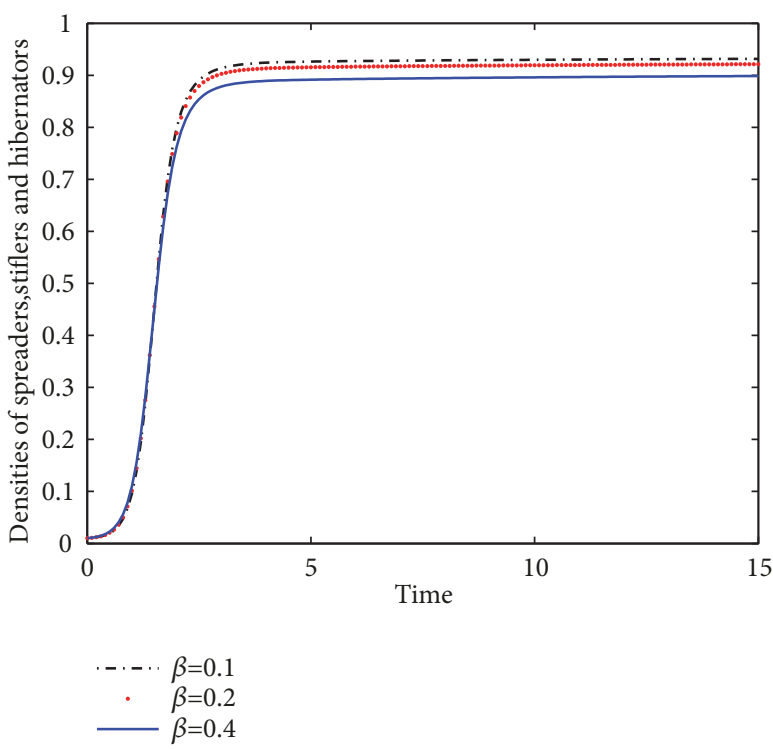

(b)

Figure 7: Numerical simulations of (3) with $\lambda=0.6, \alpha=0.2, \delta=0.2, \xi=0.1$, and $\eta=0.2$ and different refusing rates $\beta$ in the network with the Poisson degree distribution. (a) The trajectories of the densities of spreaders. (b) The trajectories of the densities of spreaders, stiflers, and hibernators.
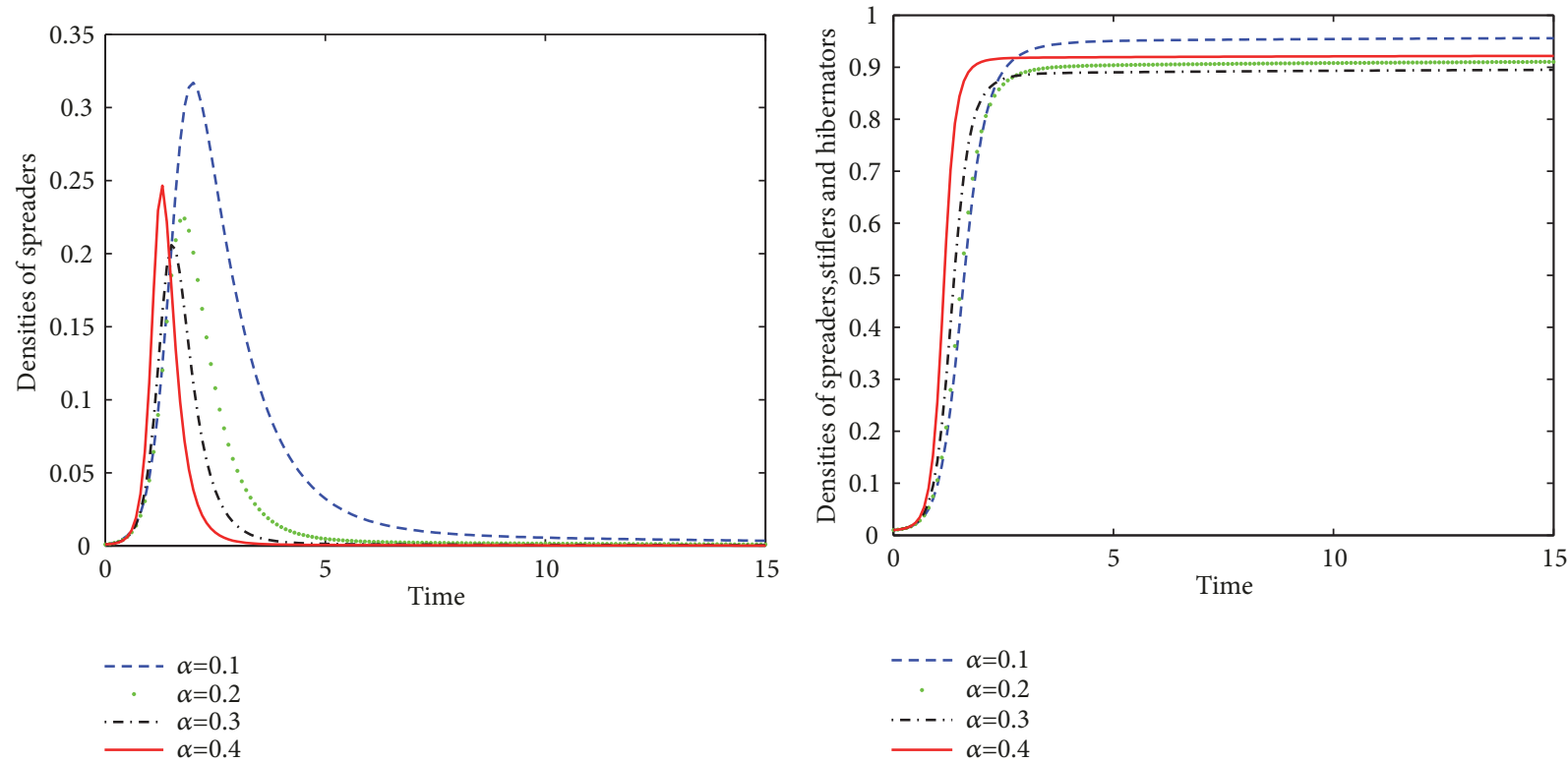

(a)

(b)

FiguRE 8: Numerical simulations of (3) with $\lambda=0.6, \beta=0.3, \delta=0.2, \xi=0.1$, and $\eta=0.2$ and different stifling rates $\alpha$ in the network with the Poisson degree distribution. (a) The trajectories of the densities of spreaders. (b) The trajectories of the densities of spreaders, stiflers, and hibernators.

on the structure of network than those under the mechanism depending on themselves.

\section{Conclusion}

This paper combines the PGF method and the pair approximation method to obtain rumor propagation model in heterogeneous networks. We obtained the following results.
(I) The comparison between numerical simulations and Monte Carlo simulations confirms the accuracy of our model.

(II) Different from the literature results, we get the threshold condition of rumor propagation, which only depends on the characteristics of the network and does not depend on the choice of the initial spreader node, which further confirms the fact that the fluctuations of the connectivity distribution cannot be neglected even for finite size systems. In fact, the 


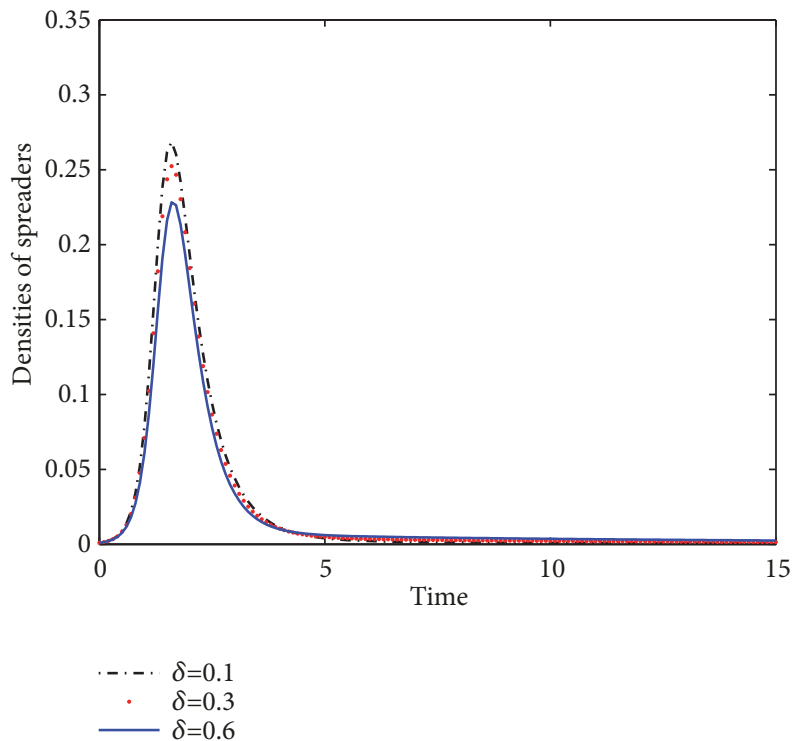

(a)

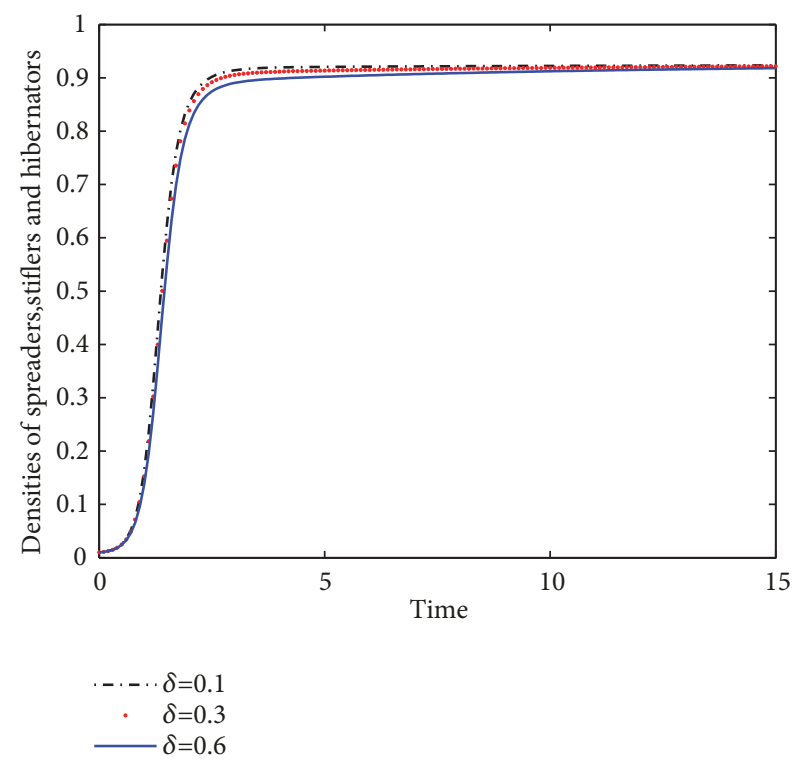

(b)

Figure 9: Numerical simulations of (3) with $\lambda=0.7, \beta=0.3, \alpha=0.2, \xi=0.1$, and $\eta=0.2$ and different $\delta$ in the network with the Poisson degree distribution. (a) The trajectories of the densities of spreaders. (b) The trajectories of the densities of spreaders, stiflers, and hibernators.

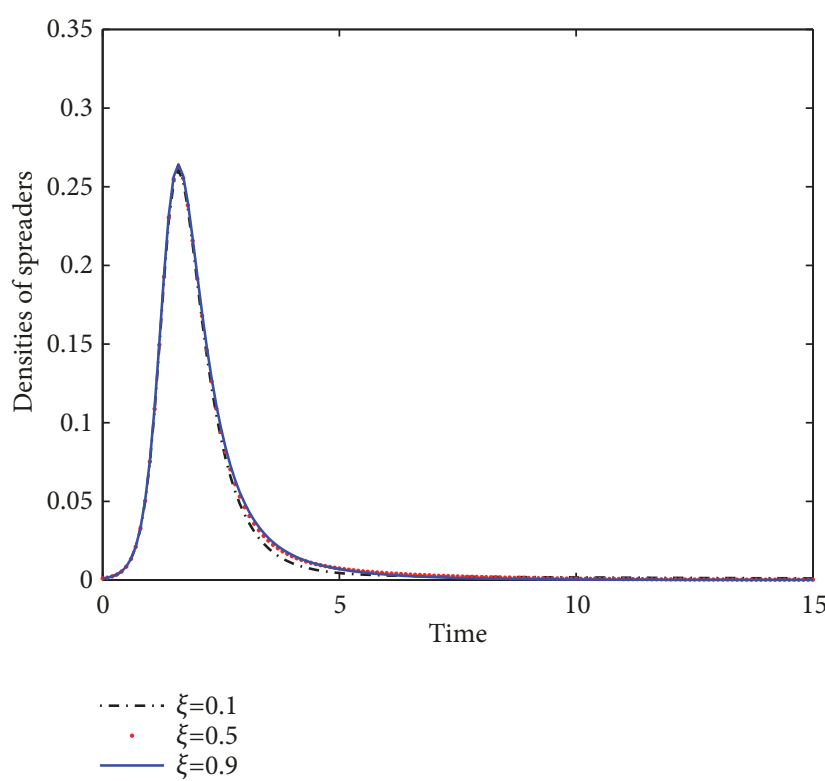

(a)

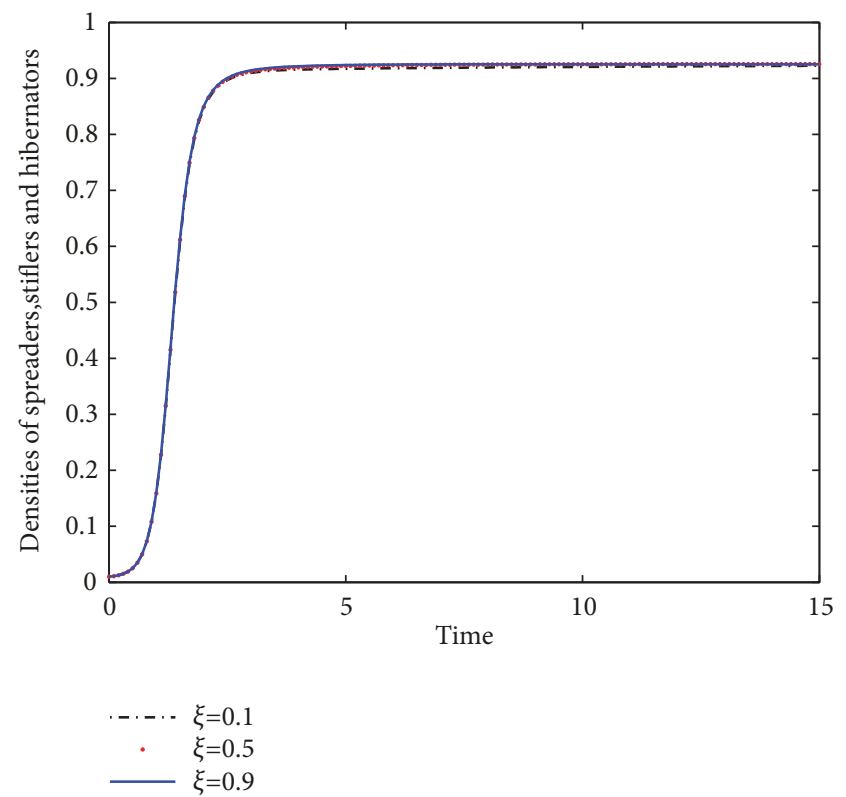

(b)

Figure 10: Numerical simulations of (3) with $\lambda=0.7, \beta=0.3, \alpha=0.2, \delta=0.2$, and $\eta=0.2$ and different $\xi$ in the network with the Poisson degree distribution. (a) The trajectories of the densities of spreaders. (b) The trajectories of the densities of spreaders, stiflers, and hibernators.

choice of the initial spreader node only affects the spread speed of rumors.

(III) The rumor recovery mechanism, different from the disease recovery mechanism, is dependent on the network. This makes its recovery rate not a constant for the rumor propagator. In the early stage of rumor spreading, the neighbors of a rumor spreader are ignorant, so the recovery time of this rumor spreader is prolonged; however, in the middle and later stage of rumor spreading, the number of the ignorant neighbors of a rumor spreader decreases, so that the rumor spreaders recover faster and faster. Therefore, this recovery mechanism will reduce the scale of transmission.

The appendix shows how the model is obtained in detail. This systemic method can be generalized to study other propagation in complex networks. 


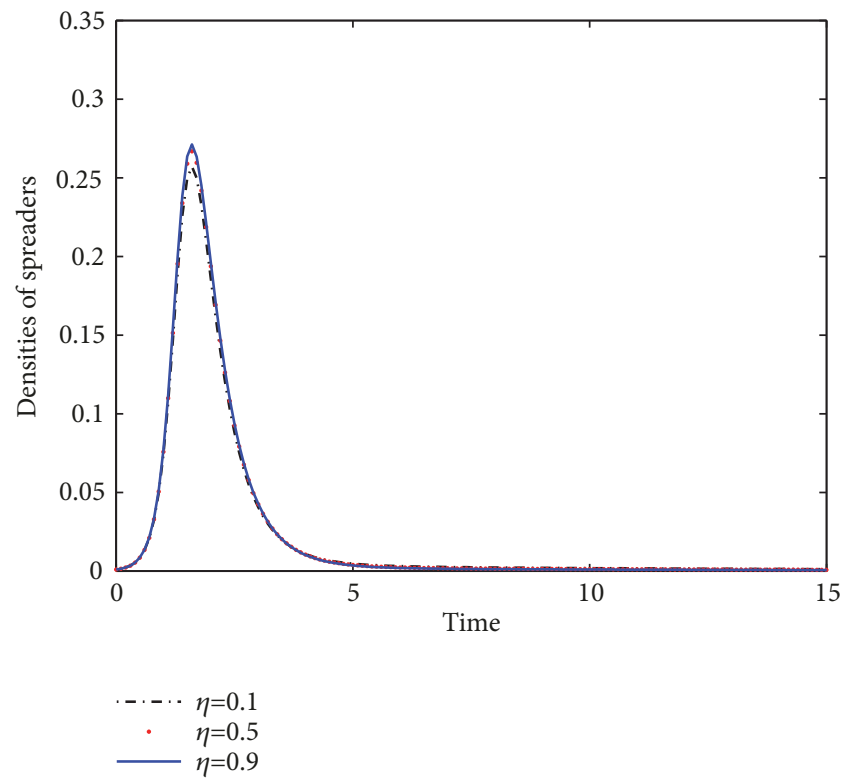

(a)

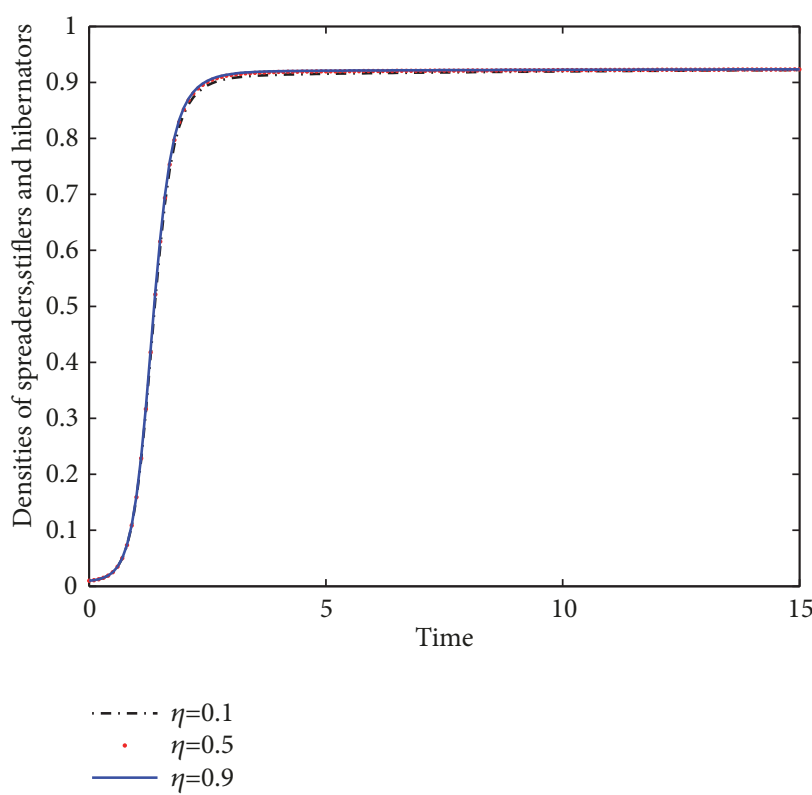

(b)

FIGURE 11: Numerical simulations of (3) with $\lambda=0.7, \beta=0.3, \alpha=0.2, \xi=0.1$, and $\delta=0.2$ and different $\eta$ in the network with the Poisson degree distribution. (a) The trajectories of the densities of spreaders. (b) The trajectories of the densities of spreaders, stiflers, and hibernators.

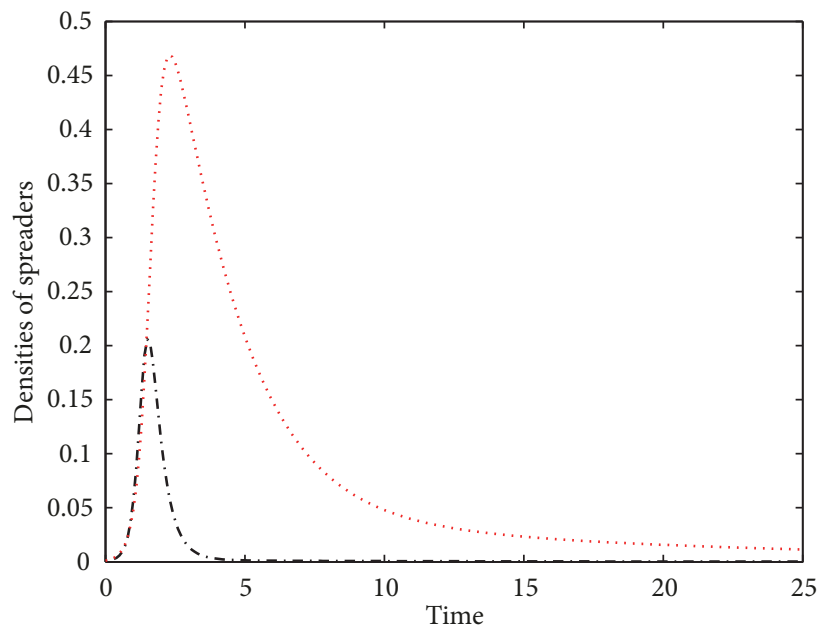

depends on network structure depends on spreader himself

(a)

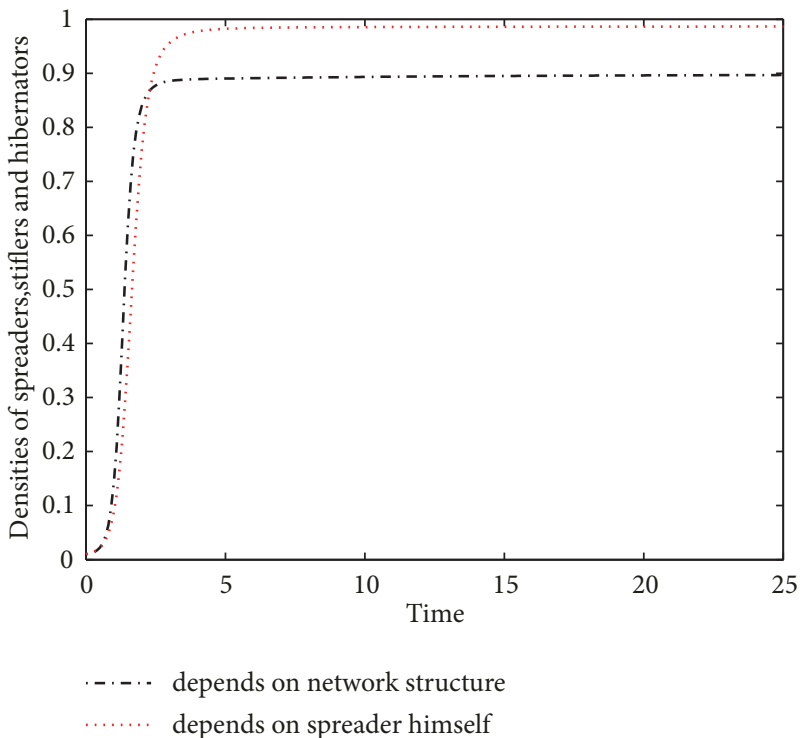

(b)

Figure 12: Numerical simulations of (3) with $\lambda=0.6, \beta=0.3, \alpha=0.3, \delta=0.2, \xi=0.1$, and $\eta=0.2$ in the networks with the Poisson degree distribution but under different recovery mechanisms. (a) The trajectories of the densities of spreaders. (b) The trajectories of the densities of spreaders, stiflers, and hibernators.

\section{Appendix}

\section{The Dynamics of SIHR Model in Random Networks}

In this section, we develop an SIHR rumor spreading model involving the variables $\theta, S_{k}, H_{k}, P_{I}^{S}, P_{I}^{H}, P_{I}^{I}, P_{H}^{S}, P_{H}^{H}$, and $P_{S}^{S}$.
The method used is similar to that [28]. To model the rumor spreading process, we need more notations given in Table 1 .

To calculate the dynamics, we choose a random ignorant ego node with degree $\mathrm{k}$ at time $\mathrm{t}$. Then there will be a set of $\mathrm{k}$ arcs $\left(\right.$ ego, alter $\left.{ }_{i}\right),(i=1,2, \ldots, k)$ corresponding to the ego node. We will assume that for each arc there will be a uniform probability $P_{I}^{S}=M_{I S} / M_{I}$ that alter $i$ is spreader. There is an 
TABLE 1: Key variables and parameters.

\begin{tabular}{lr}
\hline Symbol & Description \\
\hline$M_{A}$ & Fraction of arcs with an ego in state $A$ and an alter of any type \\
\hline$M_{A B}$ & Fraction of arcs with an ego in state $A$ and an alter in state $B$ \\
\hline$A_{B}$ & Set of arcs (ego, alter) such that node ego is in set $B$ \\
\hline$A_{A B}$ & Set of arcs (ego, alter) s.t. ego $\in A$ and alter $\in B$ \\
\hline$\delta_{A B}$ & $\begin{array}{r}\text { Represent the average degree of nodes in set } A \text {, selected with probability proportional to the number } \\
\text { of arcs to nodes in set } B, \text { not counting one arc to nodes of type } B\end{array}$ \\
\hline$\delta_{A B}(C)$ & $\begin{array}{r}\text { Set of arcs (ego, alter) but counting only arcs from ego to nodes in set } C \\
{\left[A B_{k} C\right]}\end{array}$ \\
\hline$\left[A B_{k} \cdot\right]$ & $\begin{array}{r}\text { The number of ordered triples of three nodes, the first node in state } A \text {, the second in state } B \text { with } \\
\text { degree } k, \text { and the third in state } C\end{array}$ \\
\hline
\end{tabular}

expected number $k P_{I}^{S}$ arcs such that alter is spreaders. In a time dt, an expected number $\lambda k P_{I}^{S} d t$ of these will be such that the ignorant ego received rumor becoming a spreader and $\beta k P_{I}^{S} d t$ of these will be such that the ignorant ego, who received a rumor without spreading it, becomes a stifler. Now let $I_{k}(t)$ represent the fraction of degree $\mathrm{k}$ nodes that remain ignorant at time t. Consequently, the hazard for the fraction of ignorant nodes with degree $\mathrm{k}$ becoming spreaders or stiflers at time $\mathrm{t}$ is

$$
\frac{d I_{k}(t)}{d t}=-(\lambda+\beta) k I_{k}(t) P_{I}^{S}(t)
$$

Using (A.1), we have

$$
I_{k}(t)=\left(e^{-\int_{0}^{t}(\lambda+\beta) P_{I}^{S}(\tau) d \tau}\right)^{k}
$$

For convenience, we will use the symbol $\theta$ to denote $I_{1}=$ $e^{-\int_{0}^{t}(\lambda+\beta) P_{I}^{S}(\tau) d \tau}$. From (A.2), it is clear that $I_{k}(t)=\theta^{k}$.

Given $\theta$, it is easy to determine the fraction of nodes which remain ignorants at time t. Therefore, we have

$$
I=p_{0}+p_{1} \theta+p_{2} \theta^{2}+\cdots=g(\theta) .
$$

where $g(\theta)$ is the defined probability generating function of the degree distribution $p_{k}$, which greatly simplifies subsequent equations. Similarly, the change rate of the $\theta$ can be calculated directly as follows:

$$
\dot{\theta}=-(\lambda+\beta) P_{I}^{S} \theta
$$

Since $I=g(\theta)$ from (A.3), we have

$$
\dot{I}=-(\lambda+\beta) P_{I}^{S} \theta g^{\prime}(\theta) .
$$

This does not completely specify the dynamics of $\theta$, which also depends on the variable $P_{I}^{S}$. The dynamics of $P_{I}^{S}$ is derived as follows:

$$
\dot{P_{I}^{S}}=\frac{d}{d t}\left(\frac{M_{I S}}{M_{I}}\right)=\frac{\dot{M}_{I S}}{M_{I}}-\frac{M_{I S} \dot{M}_{I}}{M_{I}^{2}} .
$$

Our goal is to put (A.6) in terms of the variables $\theta, P_{I}^{S}, P_{I}^{I} P_{I}^{H}$, and $g(\theta)$. Thus we have

$$
M_{I}=\frac{\Sigma_{k} k p_{k} I_{k}}{\sum_{k} k p_{k}}=\frac{\theta g^{\prime}(\theta)}{g^{\prime}(1)} .
$$

Using (A.7), $M_{I S}$ can easily be obtained as follows:

$$
M_{I S}=M_{I} \cdot \frac{M_{I S}}{M_{I}}=M_{I} \cdot P_{I}^{S}=\frac{P_{I}^{S} \theta g^{\prime}(\theta)}{g^{\prime}(1)} .
$$

Next consider $\dot{M}_{I}$ and $\dot{M}_{I S}$ in time dt. Firstly, $\dot{M}_{I}$ is easily placed in terms of these variable. So we have

$$
\begin{aligned}
\dot{M}_{I} & =\frac{d}{d t}\left(\frac{\theta g^{\prime}(\theta)}{g^{\prime}(1)}\right) \\
& =\frac{-(\lambda+\beta) P_{I}^{S} \theta\left[g^{\prime}(\theta)+\theta g^{\prime \prime}(\theta)\right]}{g^{\prime}(1)} .
\end{aligned}
$$

For calculating $\dot{M}_{I S}$, it requires careful consideration of the rearrangement of arcs among sets $I$ and $S$. As an ignorant contacts a spreader, the ignorant will become a spreader with a certain probability $\lambda$ or will become a stifler directly with a certain probability $\beta$. So $M_{I S}$ is reduced at a rate $(\lambda+\beta) M_{I S}$. And in time dt, $\delta S$ spreaders forget the rumor, so $M_{I S}$ is reduced at a rate $\delta M_{I S} . M_{I S}$ is also increased at the rate $\xi M_{I H}$ because $\xi H$ nodes become spreaders due to remembering spontaneously. On the other hand, in addition to its own side, the edge from the new spreader node to other ignorant or spreader neighbors will also be moved into or removed from the collection $\mathscr{A}_{I S}$. Therefore, as an ignorant has links to a spreader, the ignorant becomes a spreader. The newly spreader has on average $\delta_{I S}(I)$ arcs connected to ignorant nodes, where $\delta_{I S}(I)$ will represent the average number of arcs from ego $I$ to nodes in set $I$ not counting one arc to $S$. So $M_{I S}$ also increase at the rate $\lambda M_{I S} \delta_{I S}(I)$. As $-\dot{I}$ nodes leave set $I$ in time dt, the fraction of arcs between $I$ and $S, M_{I S}$ is reduced by the fraction of arcs from spreaders to the $-\dot{I}$ newly spreaders or stiflers. Therefore, $M_{I S}$ is reduced at the rate $-\dot{I} \delta_{I S}(S) / g^{\prime}(1)$. The quantity $M_{I S}$ is also increased; as the hibernator has links to spreaders, the stimulated hibernator 
will become a spreader and restart spreading the rumor. The new spreader will have on average $\delta_{H S}(I)$ arcs to ignorants, so $M_{I S}$ is increased at a rate $\eta M_{H S} \delta_{H S}(I)$. Meanwhile, when a spreader contacts another spreader, a hibernator, or a stifler, the initiating spreader becomes a new stifler. The newly stifler will have on average $\left(\delta_{S I}(S)+\delta_{S I}(R)+\delta_{S I}(R)\right)$ arcs to ignorants, so $M_{I S}$ is decreased at a rate $\alpha M_{I S}\left(\delta_{S I}(S)+\delta_{S I}(R)+\delta_{S I}(R)\right)$. To summarize, the derivation of the dynamics of $M_{I S}$ follows:

$$
\begin{aligned}
\dot{M}_{I S}= & (\lambda+\beta+\delta) M_{I S}+\xi M_{I H}+\lambda M_{I S} \delta_{I S}(I) \\
& +\frac{\dot{I} \delta_{I S}(S)}{g^{\prime}(1)}+\eta M_{H S} \delta_{H S}(I) \\
& -\alpha M_{I S}\left(\delta_{S I}(S)+\delta_{S I}(R)+\delta_{S I}(R)\right) \\
= & -(\lambda+\beta+\delta) M_{I S}+\xi M_{I H}+\lambda \frac{\mathscr{A}_{I S} \delta_{I S}(I)}{g^{\prime}(1) N} \\
& -(\lambda+\beta) \frac{\mathscr{A}_{I S} \delta_{I S}(S)}{g^{\prime}(1) N}+\eta \frac{\mathscr{A}_{H S} \delta_{H S}(I)}{g^{\prime}(1) N} \\
& -\alpha \frac{\mathscr{A}_{I S}}{g^{\prime}(1) N}\left(\delta_{S I}(S)+\delta_{S I}(R)+\delta_{S I}(R)\right) \\
= & -(\lambda+\beta+\delta) M_{I S}+\xi M_{I H}+\lambda \frac{\Sigma_{k}\left[I I_{k} S\right]}{g^{\prime}(1) N} \\
& -(\lambda+\beta) \frac{\sum_{k}\left[S I_{k} S\right]}{g^{\prime}(1) N}+\eta \frac{\sum_{k}\left[I H_{k} S\right]}{g^{\prime}(1) N} \\
& -\alpha \frac{\sum_{k}\left(\left[I S_{k} S\right]+\left[I S_{k} H\right]+\left[I S_{k} R\right]\right)}{g^{\prime}(1) N} .
\end{aligned}
$$

To calculate $\dot{M}_{I S}$ we need to first calculate the number of ordered triples in which the second node has degree $k$. We have

$$
\begin{aligned}
\Sigma_{k}\left[I I_{k} S\right] & =\Sigma_{k} k(k-1) N p_{k} I_{k} P_{I}^{I} P_{I}^{S} \\
& =N \theta^{2} g^{\prime \prime}(\theta) P_{I}^{I} P_{I}^{S}, \\
\Sigma_{k}\left[S I_{k} S\right] & =\Sigma_{k} k(k-1) N p_{k} I_{k}\left(P_{I}^{S}\right)^{2} \\
& =N \theta^{2} g^{\prime \prime}(\theta)\left(P_{I}^{S}\right)^{2} .
\end{aligned}
$$

Obviously, we have $\left[I S_{k} S\right]+\left[I S_{k} H\right]+\left[I S_{k} R\right]=\left[I S_{k} \cdot\right]-\left[I S_{k} I\right]$. Just like (A.11), we have

$$
\begin{aligned}
\Sigma_{k}\left[I S_{k} \cdot\right] & =\Sigma_{k} k(k-1) N p_{k} S_{k} P_{S}^{I}, \\
\Sigma_{k}\left[I S_{k} I\right] & =\Sigma_{k} k(k-1) N p_{k} S_{k}\left(P_{S}^{I}\right)^{2}, \\
\Sigma_{k}\left[I H_{k} S\right] & =\Sigma_{k} k(k-1) N p_{k} H_{k} P_{H}^{I} P_{H}^{S} .
\end{aligned}
$$

Meanwhile, $P_{S}^{I}, P_{H}^{I}$ can be represented by $P_{I}^{S}, P_{I}^{H}$ separately. So there are

$$
P_{S}^{I}=\frac{\theta g^{\prime}(\theta) P_{I}^{S}}{\sum_{k} k p_{k} S_{k}}
$$

and

$$
P_{H}^{I}=\frac{\theta g^{\prime}(\theta) P_{I}^{H}}{\Sigma_{k} k p_{k} H_{k}} .
$$

From (A.8) and (A.11) (A.14), we have

$$
\begin{gathered}
\dot{M}_{I S}=\frac{-(\lambda+\beta+\delta) \theta g^{\prime}(\theta) P_{I}^{S}}{g^{\prime}(1)}+\xi \frac{\theta g^{\prime}(\theta) P_{I}^{H}}{g^{\prime}(1)}+\lambda \\
. \frac{\theta^{2} g^{\prime \prime}(\theta) P_{I}^{I} P_{I}^{S}}{g^{\prime}(1)}-\frac{(\lambda+\beta) \theta^{2} g^{\prime \prime}(\theta)\left(P_{I}^{S}\right)^{2}}{g^{\prime}(1)}+\eta \\
. \frac{\theta g^{\prime}(\theta) \Sigma_{k} k(k-1) p_{k} H_{k} P_{I}^{H} P_{H}^{S}}{g^{\prime}(1) \Sigma_{k} k p_{k} H_{k}}-\alpha \\
. \frac{\Sigma_{k} k(k-1) p_{k} S_{k} \theta g^{\prime}(\theta) P_{I}^{S}}{\Sigma_{k} k p_{k} S_{k} g^{\prime}(1)}\left(1-\frac{\theta g^{\prime}(\theta) P_{I}^{S}}{\Sigma_{k} k p_{k} S_{k}}\right) .
\end{gathered}
$$

Now applying (A.7) (A.9) and (A.15), we solve for $\dot{P}_{I}^{S}$ in terms of $\theta$ and PGF as follows:

$$
\begin{aligned}
\dot{P_{I}^{S}=} & -(\lambda+\beta+\delta) P_{I}^{S}+\xi P_{I}^{H}+\lambda \frac{\theta g^{\prime \prime}(\theta) P_{I}^{I} P_{I}^{S}}{g^{\prime}(\theta)} \\
& +\eta \frac{\sum_{k} k(k-1) p_{k} H_{k} P_{I}^{H} P_{H}^{S}}{\Sigma_{k} k p_{k} H_{k}}+(\lambda+\beta)\left(P_{I}^{S}\right)^{2} \\
& -\alpha \frac{\sum_{k} k(k-1) p_{k} S_{k} P_{I}^{S}}{\sum_{k} k p_{k} S_{k}}\left(1-\frac{\theta g^{\prime}(\theta) P_{I}^{S}}{\sum_{k} k p_{k} S_{k}}\right) .
\end{aligned}
$$

This equation makes use of the variable $P_{I}^{I}, P_{I}^{H}, P_{H}^{S}$ which changes in time. Whose calculation is very similar to $P_{I}^{S}$. Firstly, we derive the dynamics of $P_{I}^{I}$. So we can get

$$
\dot{P_{I}^{I}}=\frac{d}{d t}\left(\frac{M_{I I}}{M_{I}}\right)=\frac{\dot{M}_{I I}}{M_{I}}-\frac{M_{I I} \dot{M}_{I}}{M_{I}^{2}}
$$

and

$$
\begin{aligned}
\dot{M}_{I I} & =-2(\lambda+\beta) M_{I S} \delta_{I S}(I) \\
& =-2(\lambda+\beta) \frac{\mathscr{A}_{I S}}{g^{\prime}(1) N} \delta_{I S}(I) \\
& =\frac{-2(\lambda+\beta) \theta^{2} g^{\prime \prime}(\theta) P_{I}^{I} P_{I}^{S}}{g^{\prime}(1)} .
\end{aligned}
$$

Therefore, we have

$$
\dot{P_{I}^{I}}=(\lambda+\beta) P_{I}^{I} P_{I}^{S}-\frac{(\lambda+\beta) \theta g^{\prime \prime}(\theta) P_{I}^{I} P_{I}^{S}}{g^{\prime}(\theta)} .
$$

Similarly, applying the above mathematical method, the calculation of $P_{I}^{H}$ can be derived. So we have 


$$
\dot{P_{I}^{H}}=\frac{d}{d t}\left(\frac{M_{I H}}{M_{I}}\right)=\frac{\dot{M_{I H}}}{M_{I}}-\frac{M_{I H} \dot{M_{I}}}{M_{I}^{2}}
$$

and

$$
\begin{aligned}
\dot{M_{I H}=} & -(\lambda+\beta) M_{I S} \delta_{I S}(H)-\xi M_{I H}+\delta M_{I S} \\
& -\eta M_{H I} \delta_{H I}(S) \\
= & -\frac{(\lambda+\beta) \theta^{2} g^{\prime \prime}(\theta) P_{I}^{S} P_{I}^{H}}{g^{\prime}(1)}-\frac{\xi \theta g^{\prime}(\theta) P_{I}^{H}}{g^{\prime}(1)} \\
& +\frac{\delta \theta g^{\prime}(\theta) P_{I}^{S}}{g^{\prime}(1)}-\frac{\eta \Sigma_{k} k(k-1) p_{k} H_{k} P_{H}^{I} P_{H}^{S}}{g^{\prime}(1)} .
\end{aligned}
$$

$$
\begin{aligned}
\dot{H}_{k} & =\delta S_{k}-\xi H_{k}-\eta k H_{k} P_{H}^{S}, \\
\dot{M}_{H} & =\frac{\Sigma_{k} k p_{k} \dot{H}_{k}}{g^{\prime}(1)}=\frac{\delta \Sigma_{k} k p_{k} S_{k}-\xi \Sigma_{k} k p_{k} H_{k}-\eta \Sigma_{k} k(k-1) p_{k} H_{k} P_{H}^{S}-\eta \Sigma_{k} k p_{k} H_{k} P_{H}^{S}}{g^{\prime}(1)},
\end{aligned}
$$

From (A.20) and (A.21), we have

$$
\begin{aligned}
\dot{P_{I}^{H}}= & -\xi P_{I}^{H}+\delta P_{I}^{S}+(\lambda+\beta) P_{I}^{S} P_{I}^{H} \\
& -\frac{\eta \Sigma_{k} k(k-1) p_{k} H_{k} P_{I}^{H} P_{H}^{S}}{\Sigma_{k} k p_{k} H_{k}} .
\end{aligned}
$$

Now, it is necessary to determine the derivation of $P_{H}^{S}$ to complete the model. In the same way, we have

$$
\dot{P_{H}^{S}}=\frac{d}{d t}\left(\frac{M_{H S}}{M_{H}}\right)=\frac{\dot{M_{H S}}}{M_{H}}-\frac{M_{H S} \dot{M}_{H}}{M_{H}^{2}},
$$

For calculating $\dot{P_{H}^{S}}$, we need to obtain the following: and

$$
\begin{aligned}
M_{H S}= & \delta M_{S S}+\xi M_{H H}+\lambda M_{I H} \delta_{I H}(S) \\
& +\eta M_{H H} \delta_{H H}(S)-(\delta+\xi+\eta+\alpha) M_{H S} \\
& -\eta M_{H S} \delta_{H S}(S) \\
& -\alpha M_{S H}\left(\delta_{S H}(S)+\delta_{S H}(H)+\delta_{S H}(R)\right) \\
= & \delta \frac{\sum_{k} k p_{k} S_{k} P_{S}^{S}}{g^{\prime}(1)}+\xi \frac{\Sigma_{k} k p_{k} H_{k} P_{H}^{H}}{g^{\prime}(1)} \\
& +\lambda \frac{\theta^{2} g^{\prime \prime}(\theta) P_{I}^{S} P_{I}^{H}}{g^{\prime}(1)} \\
& +\eta \frac{\sum_{k} k(k-1) p_{k} H_{k}}{g^{\prime}(1)} P_{H}^{H} P_{H}^{S} \\
& -(\delta+\xi+\eta+\alpha) \frac{\Sigma_{k} k p_{k} H_{k} P_{H}^{S}}{g^{\prime}(1)} \\
& -\eta \frac{\sum_{k} k(k-1) p_{k} H_{k}\left(P_{H}^{S}\right)^{2}}{g^{\prime}(1)} \\
& -\alpha \frac{\sum_{k} k(k-1) p_{k} S_{k} P_{S}^{H}}{g^{\prime}(1)}\left(1-P_{S}^{I}\right) .
\end{aligned}
$$

From (A.23) (A.26), we have

$$
\begin{aligned}
\dot{P_{H}^{S}}= & \delta \frac{\Sigma_{k} k p_{k} S_{k}\left(P_{S}^{S}-P_{H}^{S}\right)}{\Sigma_{k} k p_{k} H_{k}}+\xi P_{H}^{H} \\
& +\lambda \frac{\theta^{2} g^{\prime \prime}(\theta) P_{I}^{S} P_{I}^{H}}{\Sigma_{k} k p_{k} H_{k}}
\end{aligned}
$$

$$
\begin{aligned}
& +\eta \frac{\Sigma_{k} k(k-1) p_{k} H_{k}}{\Sigma_{k} k p_{k} H_{k}} P_{H}^{H} P_{H}^{S}-(\delta+\eta+\alpha) P_{H}^{S} \\
& -\alpha \frac{\Sigma_{k} k(k-1) p_{k} S_{k} P_{H}^{S}}{\Sigma_{k} k p_{k} S_{k}}\left(1-\frac{\theta g^{\prime}(\theta) P_{I}^{S}}{\Sigma_{k} k p_{k} S_{k}}\right) \\
& +\eta\left(P_{H}^{S}\right)^{2} .
\end{aligned}
$$

This equation makes use of the variable $P_{S}^{S}, P_{H}^{H}$ which changes in time. Deriving the dynamics of this variable will complete the model. So we have the following results. Next consider $\dot{P_{H}^{H}}$; we have

$$
\dot{P_{H}^{H}}=\frac{d}{d t}\left(\frac{M_{H H}}{M_{H}}\right)=\frac{\dot{M_{H H}}}{M_{H}}-\frac{M_{H H} \dot{M}_{H}}{M_{H}^{2}} .
$$

For the goal of $M_{H H}$, we must arrange the change of arcs $H H$; we have

$$
\begin{aligned}
M_{H H}^{\cdot}= & 2 \delta M_{S H}-2 \xi M_{H H}-2 \eta M_{H S} \delta_{H S}(H) \\
= & 2 \delta \frac{\sum_{k} k p_{k} H_{k} P_{H}^{S}}{g^{\prime}(1)}-2 \xi \frac{\Sigma_{k} k p_{k} H_{k} P_{H}^{H}}{g^{\prime}(1)} \\
& -2 \eta \frac{\sum_{k} k(k-1) p_{k} H_{k} P_{H}^{H} P_{H}^{S}}{g^{\prime}(1)} .
\end{aligned}
$$

From (A.25), (A.28), and (A.29), we have

$$
\begin{aligned}
\dot{P_{H}^{H}}= & 2 \delta P_{H}^{S}-\xi P_{H}^{H}-\eta \frac{\Sigma_{k} k(k-1) p_{k} H_{k} P_{H}^{H} P_{H}^{S}}{\Sigma_{k} k p_{k} H_{k}} \\
& -\delta \frac{\Sigma_{k} k p_{k} S_{k} p_{H}^{H}}{\Sigma_{k} k p_{k} H_{k}}+\eta P_{H}^{S} P_{H}^{H} .
\end{aligned}
$$


Finally, it is necessary to determine the time derivative of $\dot{P}_{S}^{S}$.

$$
\dot{P_{S}^{S}}=\frac{d}{d t}\left(\frac{M_{S S}}{M_{S}}\right)=\frac{\dot{M}_{S S}}{M_{S}}-\frac{M_{S S} \dot{M}_{S}}{M_{S}{ }^{2}} .
$$

Next consider $\dot{M}_{S}$ in time dt. Because $\dot{M}_{S}=\Sigma_{k} k p_{k} \dot{S}_{k} / g^{\prime}(1)$, we have

$$
\begin{aligned}
\dot{S_{k}}= & -\delta S_{k}+\xi H_{k}+\lambda k I_{k} P_{I}^{S}+\eta k H_{k} P_{H}^{S} \\
& -\alpha\left(k S_{k}-k S_{k} P_{S}^{I}\right) .
\end{aligned}
$$

From (A.32), we have

$$
\begin{aligned}
\dot{M}_{S}= & \lambda \frac{\sum_{k} k^{2} p_{k} I_{k} P_{I}^{S}}{g^{\prime}(1)}-\delta \frac{\sum_{k} k p_{k} S_{k}}{g^{\prime}(1)}+\xi \frac{\sum_{k} k p_{k} H_{k}}{g^{\prime}(1)} \\
& +\eta \frac{\sum_{k} k^{2} p_{k} H_{k} P_{H}^{S}}{g^{\prime}(1)}-\alpha \frac{\sum_{k} k^{2} p_{k} S_{k}\left(1-P_{S}^{I}\right)}{g^{\prime}(1)} .
\end{aligned}
$$

For the goal of $\dot{M}_{S S}$, we must arrange the change of arcs SS. So we have

$$
\begin{aligned}
& \dot{M}_{S S}=-2 \delta M_{S S}+2 \xi M_{H S}+2 \lambda M_{I S} \delta_{I S}(S)+2 \lambda M_{I S} \\
&+2 \eta M_{H S}+2 \eta M_{H S} \delta_{H S}(S) \\
&-2 \alpha M_{S S}\left(\delta_{S S}(S)+\delta_{S S}(H)+\delta_{S S}(R)\right) \\
&=-2 \delta \frac{\sum_{k} k p_{k} S_{k} P_{S}^{S}}{g^{\prime}(1)}+2 \xi \frac{\Sigma_{k} k p_{k} H_{k} P_{H}^{S}}{g^{\prime}(1)} \\
&+2 \lambda \frac{\theta^{2} g^{\prime \prime}(\theta)\left(P_{I}^{S}\right)^{2}}{g^{\prime}(1)}+2 \lambda \frac{\theta g^{\prime}(\theta) P_{I}^{S}}{g^{\prime}(1)} \\
&+2 \eta \frac{\sum_{k} k p_{k} H_{k} P_{H}^{S}}{g^{\prime}(1)} \\
&+2 \eta \frac{\sum_{k} k(k-1) p_{k} H_{k}\left(P_{H}^{S}\right)^{2}}{g^{\prime}(1)} \\
&-2 \alpha \frac{\sum_{k} k(k-1) p_{k} S_{k} P_{S}^{S}\left(1-P_{S}^{I}\right)}{g^{\prime}(1)} \\
&-2 g_{k} S_{k} P_{S}^{S} \\
&
\end{aligned}
$$

From (A.32) (A.34), we have

$$
\begin{aligned}
\dot{P_{S}^{S}=} & (-\alpha-\delta) P_{S}^{S} \\
& +\frac{\sum_{k} k p_{k} H_{k}}{\sum_{k} k p_{k} S_{k}}\left(2 \xi P_{H}^{S}-\xi P_{S}^{S}+2 \eta P_{H}^{S}-\eta P_{H}^{S} P_{S}^{S}\right) \\
& +\lambda \frac{\theta^{2} g^{\prime \prime}(\theta)}{\sum_{k} k p_{k} S_{k}} P_{I}^{S}\left(2 P_{I}^{S}-P_{S}^{S}\right) \\
& +\lambda \frac{\theta g^{\prime}(\theta)}{\sum_{k} k p_{k} S_{k}} P_{I}^{S}\left(2-P_{S}^{S}\right) \\
& +\eta \frac{\sum_{k} k(k-1) p_{k} H_{k}}{\sum_{k} k p_{k} S_{k}} P_{H}^{S}\left(2 P_{H}^{S}-P_{S}^{S}\right) \\
& -\alpha \frac{\sum_{k} k(k-1) p_{k} S_{k} P_{S}^{S}}{\sum_{k} k p_{k} S_{k}} \\
& +\alpha \frac{\theta g^{\prime}(\theta) \sum_{k} k(k-1) p_{k} S_{k} P_{S}^{S} P_{I}^{S}}{\left(\sum_{k} k p_{k} S_{k}\right)^{2}} \\
& -\alpha \frac{\theta g^{\prime}(\theta) P_{I}^{S} P_{S}^{S}}{\sum_{k} k p_{k} S_{k}} .
\end{aligned}
$$

\section{Data Availability}

The data used to support the findings of this study are available from the corresponding author upon request.

\section{Conflicts of Interest}

The authors declare that no conflicts of interest exist with regard to the publication of this paper.

\section{Acknowledgments}

The research is supported by the National Natural Science Foundations of China under Grant snos. 11571210, 11331009, 11501339, 11101251, 11001157, 11471197, and 11701348 and the Youth Science Foundation of Shanxi Province no. 20100210011.

\section{References}

[1] K. Kawachi, M. Seki, H. Yoshida, Y. Otake, K. Warashina, and H. Ueda, "A rumor transmission model with various contact interactions," Journal of Theoretical Biology, vol. 253, no. 1, pp. 55-60, 2008

[2] Z. L. Zhang and Z. Q. Zhang, "An interplay model for rumour spreading and emergency development," Physica A: Statistical Mechanics and its Applications, vol. 388, no. 19, pp. 4159-4166, 2009.

[3] M. Kosfeld, "Rumours and markets," Journal of Mathematical Economics, vol. 41, no. 6, pp. 646-664, 2005.

[4] J. Kostka, Y. A. Oswald, and R. Wattenhofer, "Word of mouth: rumor dissemination in social networks," in Structural Information and Communication Complexity, vol. 5058 of Lecture Notes 
in Computer Science, pp. 185-196, Springer, Berlin, Germany, 2008.

[5] D. Liu and X. Chen, "Rumor propagation in online social networks like Twitter - A simulation study," in Proceedings of the 3rd International Conference on Multimedia Information Networking and Security, MINES 2011, pp. 278-282, China, November 2011.

[6] D. J. Daley and D. G. Kendall, "Epidemics and rumours," Nature, vol. 204, no. 4963, p. 1118, 1964.

[7] D. J. Daley and D. G. Kendall, "Stochastic rumours," Journal of the Institute of Mathematics and Its Applications, vol. 1, pp. 4255, 1965.

[8] D. P. Maki and M. Thompson, Mathematical Models and Applications: With Emphasis on the Social, Life, and Management Sciences, Prentice Hall, Englewood Cliffs, NJ, USA, 1973.

[9] L.-L. Xia, G.-P. Jiang, B. Song, and Y.-R. Song, "Rumor spreading model considering hesitating mechanism in complex social networks," Physica A: Statistical Mechanics and its Applications, vol. 437, pp. 295-303, 2015.

[10] Y. Zan, J. Wu, P. Li, and Q. Yu, "SICR rumor spreading model in complex networks: counterattack and self-resistance," Physica A: Statistical Mechanics and its Applications, vol. 405, pp. 159170, 2014.

[11] R. Jie, J. Qiao, G. Xu, and Y. Meng, "A study on the interaction between two rumors in homogeneous complex networks under symmetric conditions," Physica A: Statistical Mechanics and its Applications, vol. 454, pp. 129-142, 2016.

[12] J. Gu, W. Li, and X. Cai, "The effect of the forget-remember mechanism on spreading," The European Physical Journal B, vol. 62, no. 2, pp. 247-255, 2008.

[13] L. J. Zhao, J. J. Wang, Y. C. Chen, Q. Wang, J. Cheng, and H. Cui, "SIHR rumor spreading model in social networks," Physica A: Statistical Mechanics and its Applications, vol. 391, no. 7, pp. 2444-2453, 2012.

[14] C. Lefevre and P. Picard, "Distribution of the final extent of a rumour process," Journal of Applied Probability, vol. 31, no. 1, pp. 244-249, 1994.

[15] M. E. J. Newman, S. Forrest, and J. Balthrop, "Email networks and the spread of computer viruses," Physical Review E: Statistical, Nonlinear, and Soft Matter Physics, vol. 66, no. 3, Article ID 035101, 4 pages, 2002.

[16] D. H. Zanette, "Critical behavior of propagation on small-world networks," Physical Review E: Statistical, Nonlinear, and Soft Matter Physics, vol. 64, no. 5, Article ID 050901, 2001.

[17] D. H. Zanette, "Dynamics of rumor propagation on small-world networks," Physical Review E: Statistical, Nonlinear, and Soft Matter Physics, vol. 65, no. 1, Article ID 041908, 9 pages, 2002.

[18] L. Buzna, K. Peters, and D. Helbing, "Modelling the dynamics of disaster spreading in networks," Physica A: Statistical Mechanics and its Applications, vol. 363, no. 1, pp. 132-140, 2006.

[19] M. Nekovee, Y. Moreno, G. Bianconi, and M. Marsili, “Theory of rumour spreading in complex social networks," Physica A: Statistical Mechanics and its Applications, vol. 374, no. 1, pp. 457470, 2007.

[20] Y. Moreno, M. Nekovee, and A. F. Pacheco, "Dynamics of rumor spreading in complex networks," Physical Review E: Statistical, Nonlinear, and Soft Matter Physics, vol. 69, no. 6, Article ID 066130, 2004.

[21] L. Zhao, X. Qiu, X. Wang, and J. Wang, "Rumor spreading model considering forgetting and remembering mechanisms in inhomogeneous networks," Physica A: Statistical Mechanics and its Applications, vol. 392, no. 4, pp. 987-994, 2013.
[22] F. Roshani and Y. Naimi, "Effects of degree-biased transmission rate and nonlinear infectivity on rumor spreading in complex social networks," Physical Review E: Statistical, Nonlinear, and Soft Matter Physics, vol. 85, no. 3, Article ID 036109, 2012.

[23] L. Zhu and Y. Wang, "Rumor spreading model with noise interference in complex social networks," Physica A: Statistical Mechanics and its Applications, vol. 469, pp. 750-760, 2017.

[24] Y.-Q. Wang, X.-Y. Yang, Y.-L. Han, and X.-A. Wang, "Rumor spreading model with trust mechanism in complex social networks," Communications in Theoretical Physics, vol. 59, no. 4, pp. 510-516, 2013.

[25] J. Wang, L. Zhao, and R. Huang, "SIRaRu rumor spreading model in complex networks," Physica A: Statistical Mechanics and its Applications, vol. 398, no. 15, pp. 43-55, 2014.

[26] Q. Liu, T. Li, and M. Sun, "The analysis of an SEIR rumor propagation model on heterogeneous network," Physica A: Statistical Mechanics and its Applications, vol. 469, pp. 372-380, 2017.

[27] C. Wan, T. Li, Y. Wang, and X. Liu, "Rumor spreading of a SICS model on complex social networks with counter mechanism," Open Access Library Journal, vol. 03, no. 07, pp. 1-11, 2016.

[28] E. Volz, "SIR dynamics in random networks with heterogeneous connectivity," Journal of Mathematical Biology, vol. 56, no. 3, pp. 293-310, 2008.

[29] J. Li, J. Wang, and Z. Jin, "SIR dynamics in random networks with communities," Journal of Mathematical Biology, vol. 77, no. 4, pp. 1117-1151, 2018.

[30] J. Li, W. Li, and Z. Jin, "The epidemic model based on the approximation for third-order motifs on networks," Mathematical Biosciences, vol. 297, pp. 12-26, 2018.

[31] M. Molloy and B. Reed, "A critical point for random graphs with a given degree sequence," Random Structures \& Algorithms, vol. 6, no. 2-3, pp. 161-179, 1995. 


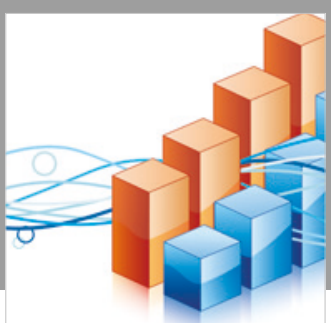

Advances in

Operations Research

\section{-n-m}
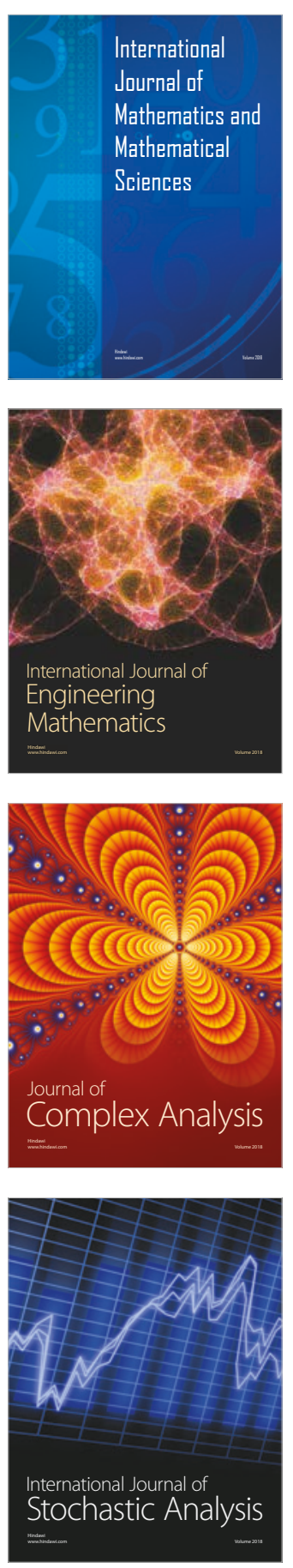
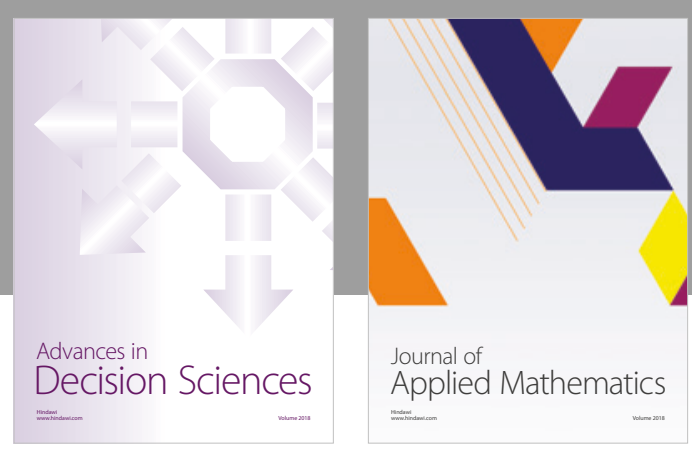

Journal of

Applied Mathematics
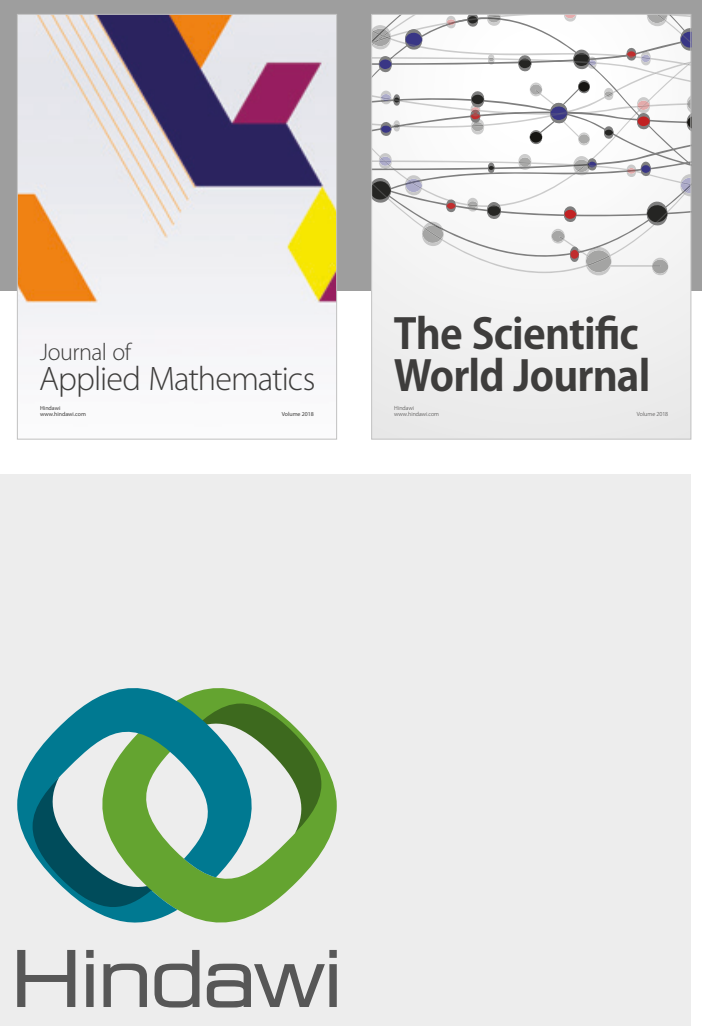

Submit your manuscripts at

www.hindawi.com

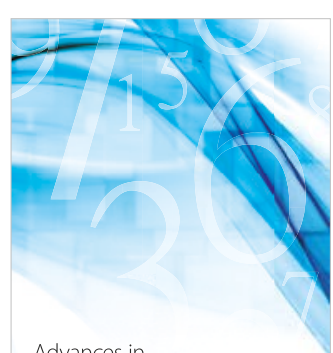

Advances in
Numerical Analysis
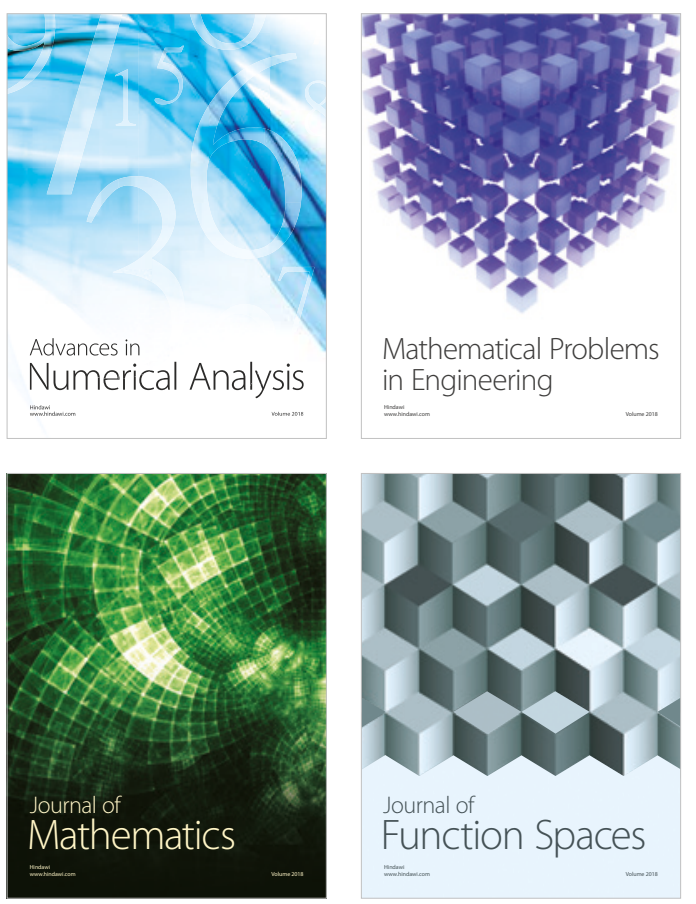

Mathematical Problems in Engineering

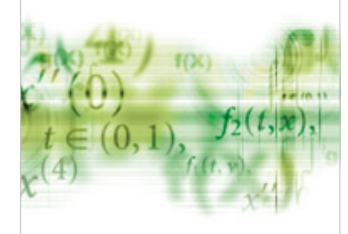

International Journal of

Differential Equations

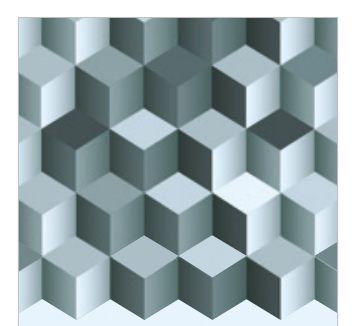

Journal of

Function Spaces
The Scientific

World Journal

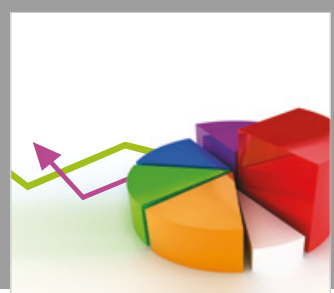

Journal of

Probability and Statistics
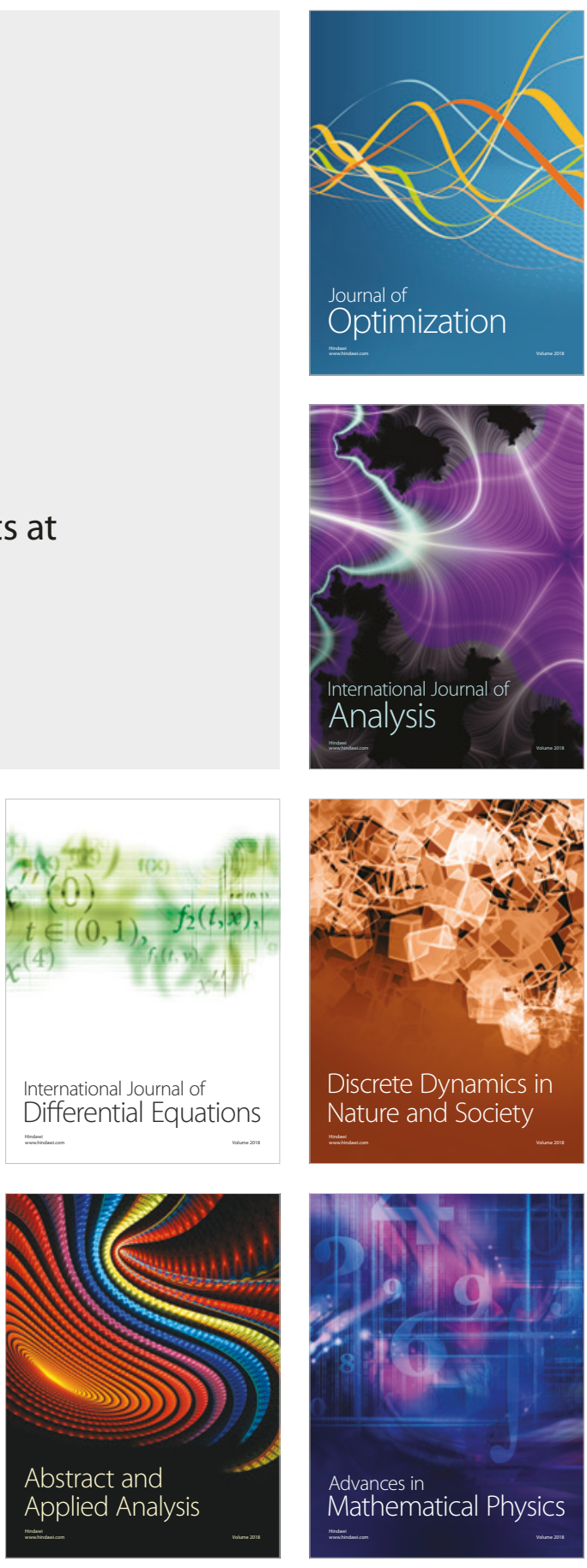